\title{
QUASI-PERIODIC SOLUTIONS OF COMPLETELY RESONANT FORCED WAVE EQUATIONS
}

\author{
MASSIMILIANO BERTI, MICHELA PROCESI
}

\begin{abstract}
We prove existence of quasi-periodic solutions with two frequencies of completely resonant, periodically forced nonlinear wave equations with periodic spatial boundary conditions. We consider both the cases the forcing frequency is: (Case A) a rational number and (Case B) an irrational number.
\end{abstract}

Keywords: Nonlinear Wave Equation, Quasi-Periodic Solutions, Variational Methods, Lyapunov-Schmidt reduction, Infinite Dimensional Hamiltonian Systems. ${ }^{1}$

2000AMS subject classification: 35L05, 37K50, 58E05.

\section{Introduction}

We prove existence of small amplitude quasi-periodic solutions for completely resonant forced nonlinear wave equations like

$$
\left\{\begin{array}{l}
v_{t t}-v_{x x}+f\left(\omega_{1} t, v\right)=0 \\
v(t, x)=v(t, x+2 \pi)
\end{array}\right.
$$

where the nonlinear forcing term

$$
f\left(\omega_{1} t, v\right)=a\left(\omega_{1} t\right) v^{2 d-1}+O\left(v^{2 d}\right), \quad d>1, d \in \mathbb{N}^{+}
$$

is $2 \pi / \omega_{1}$-periodic in time. We shall consider both the cases

- A) the forcing frequency $\omega_{1} \in \mathbb{Q}$

- B) the forcing frequency $\omega_{1} \in \mathbb{R} \backslash \mathbb{Q}$.

Existence of periodic solutions for completely resonant forced wave equations was first proved in the pioneering papers [R1], R2] (with Dirichlet boundary conditions) if the forcing frequency is a rational number $\left(\omega_{1}=1\right.$ in $\left.\left.[\mathrm{R} 1]-\mathrm{R} 2\right]\right)$. This requires to solve an infinite dimensional bifurcation equation which lacks compactness property; see $[\mathrm{BN},[\mathrm{C}], \mathrm{BBi}-\mathrm{BBi1}$ and references therein for other results. If the forcing frequency is an irrational number existence of periodic solutions has been proved in $[\mathrm{PY}]-[\mathrm{Mc}]$ : here the bifurcation equation is trivial but a "small divisors problem" appears.

To prove existence of small amplitude quasi-periodic solutions for completely resonant PDE's like (1.1) one generally has to deal with a small divisor problem as well; however the main difficulty is to understand from which solutions of the linearized equation at $v=0$,

$$
v_{t t}-v_{x x}=0
$$

Massimiliano Berti, SISSA, via Beirut 2-4, Trieste, Italy, berti@sissa.it . Michela Procesi, Università di Roma 3, Largo S. Leonardo Murialdo, Roma, Italy, procesi@mat.uniroma3.it .

${ }^{1}$ Supported by M.I.U.R. "Variational Methods and Nonlinear Differential Equations". 
quasi-periodic solutions branch-off: such linearized equation possesses only $2 \pi$-periodic solutions $q_{+}(t+x)+q_{-}(t-x)$ where $q_{+}(\cdot), q_{-}(\cdot)$ are $2 \pi$-periodic (completely resonant PDE).

Here is the main difference w.r.t non-resonant PDE's for which a developed existence theory of periodic and quasi-periodic solutions has been established, see e.g. [K], Wa], [CW], $\mathrm{P}$, [B1] and references therein.

For completely resonant autonomous PDE's, existence of periodic solutions has been proved in LS, [BP, BB1, BB2, BB3, GMP, GP, and quasi-periodic solutions with 2-frequencies have been recently obtained in $\mathrm{P} 1$ - $\mathrm{P} 2$ for the specific nonlinearities $f=u^{3}+O\left(u^{5}\right)$. Here the bifurcation equation is solved by ODE methods.

In this paper we prove existence of quasi-periodic solutions with two frequencies $\omega_{1}$, $\omega_{2}$ for the completely resonant forced equation (1.1) in both the two cases: Case $A$ ): $\omega_{1} \in \mathbb{Q}$; Case $\left.B\right): \omega_{1} \in \mathbb{R} \backslash \mathbb{Q}$.

The more interesting case is $\omega_{1} \in \mathbb{Q}$ (case A) when the forcing frequency $\omega_{1}$ enters in resonance with the linear frequency 1 . To find out from which solutions of the linearized equation quasi-periodic solutions of (1.1) branch-off, requires to solve an infinite dimensional bifurcation equation which can not be solved in general by ODE techniques (it is a system of integro-differential equations). However, exploiting the variational nature of equation (1.1) like in BB1]-[BB2, the bifurcation problem can be reduced to finding critical points of a suitable action functional which, in this case, possesses the infinite dimensional linking geometry $[\mathrm{BR}]$.

1.1. Main results. We look for quasi-periodic solutions $v(t, x)$ of equation (1.1) of the form

$$
\left\{\begin{array}{l}
v(t, x)=u\left(\omega_{1} t, \omega_{2} t+x\right) \\
u\left(\varphi_{1}+2 k_{1} \pi, \varphi_{2}+2 k_{2} \pi\right)=u\left(\varphi_{1}, \varphi_{2}\right), \quad \forall k_{1}, k_{2} \in \mathbb{Z}
\end{array}\right.
$$

with frequencies

$$
\omega=\left(\omega_{1}, \omega_{2}\right)=\left(\omega_{1}, 1+\varepsilon\right),
$$

imposing the frequency $\omega_{2}=1+\varepsilon$ to be close to the linear frequency 1 .

Writing $\partial_{t t}-\partial_{x x}=\left(\partial_{t}-\partial_{x}\right) \circ\left(\partial_{t}+\partial_{x}\right)$ we get

$$
\left[\omega_{1} \partial_{\varphi_{1}}+\left(\omega_{2}-1\right) \partial_{\varphi_{2}}\right] \circ\left[\omega_{1} \partial_{\varphi_{1}}+\left(\omega_{2}+1\right) \partial_{\varphi_{2}}\right] u+f\left(\varphi_{1}, u\right)=0
$$

and therefore

$$
\left[\omega_{1}^{2} \partial_{\varphi_{1}}^{2}+\left(\omega_{2}^{2}-1\right) \partial_{\varphi_{2}}^{2}+2 \omega_{1} \omega_{2} \partial_{\varphi_{1}} \partial_{\varphi_{2}}\right] u(\varphi)+f\left(\varphi_{1}, u\right)=0 .
$$

We assume that the forcing term $f: \mathbb{T} \times \mathbb{R} \rightarrow \mathbb{R}$

$$
f\left(\varphi_{1}, u\right)=a_{2 d-1}\left(\varphi_{1}\right) u^{2 d-1}+O\left(u^{2 d}\right), \quad d \in \mathbb{N}^{+}, d>1
$$

is analytic in $u$ but has only finite regularity in $\varphi_{1}$. More precisely

- (H) $f\left(\varphi_{1}, u\right):=\sum_{k=2 d-1}^{\infty} a_{k}\left(\varphi_{1}\right) u^{k}, d \in \mathbb{N}^{+}, d>1$ and the coefficients $a_{k}\left(\varphi_{1}\right) \in$ $H^{1}(\mathbb{T})$ verify, for some $r>0, \sum_{k=2 d-1}^{\infty}\left|a_{k}\right|_{H^{1}} r^{k}<\infty$. The function $f\left(\varphi_{1}, u\right)$ is not identically constant in $\varphi_{1}$. 
We look for solutions $u$ of (1.4) in the Banach space ${ }^{2}$

$$
\mathcal{H}_{\sigma, s}:=\left\{u(\varphi)=\sum_{l \in \mathbb{Z}^{2}} \hat{u}_{l} e^{\mathrm{i} l \cdot \varphi}: \hat{u}_{l}^{*}=\hat{u}_{-l} \text { and }|u|_{\sigma, s}:=\sum_{l \in \mathbb{Z}^{2}}\left|\hat{u}_{l}\right| e^{\left|l_{2}\right| \sigma}\left[l_{1}\right]^{s}<+\infty\right\}
$$

where $\left[l_{1}\right]:=\max \left\{\left|l_{1}\right|, 1\right\}$ and $\sigma>0, s \geq 0$.

The space $\mathcal{H}_{\sigma, s}$ is a Banach algebra with respect to multiplications of functions (see Lemma 4.1 in the Appendix), namely

$$
u_{1}, u_{2} \in \mathcal{H}_{\sigma, s} \quad \Longrightarrow \quad u_{1} u_{2} \in \mathcal{H}_{\sigma, s} \quad \text { and } \quad\left|u_{1} u_{2}\right|_{\sigma, s} \leq C\left|u_{1}\right|_{\sigma, s}\left|u_{2}\right|_{\sigma, s} .
$$

We shall prove the following Theorems.

Theorem A. Let $\omega_{1}=n / m \in \mathbb{Q}$. Assume that $f$ satisfies assumption $(\mathbf{H})$ and $a_{2 d-1}\left(\varphi_{1}\right) \neq 0, \forall \varphi_{1} \in \mathbb{T}$. Let $\mathcal{B}_{\gamma}$ be the uncountable ${ }^{3}$ zero-measure Cantor set

$$
\mathcal{B}_{\gamma}:=\left\{\varepsilon \in\left(-\varepsilon_{0}, \varepsilon_{0}\right):\left|l_{1}+\varepsilon l_{2}\right|>\frac{\gamma}{\left|l_{2}\right|}, \forall l_{1}, l_{2} \in \mathbb{Z} \backslash\{0\}\right\}
$$

where $0<\gamma<1 / 6$.

There exist constants $\bar{\sigma}>0, \bar{s}>2, \bar{\varepsilon}>0, \bar{C}>0$, such that $\forall \varepsilon \in \mathcal{B}_{\gamma},|\varepsilon| \gamma^{-1} \leq \bar{\varepsilon} / m^{2}$, there exists a classical solution $u(\varepsilon, \varphi) \in \mathcal{H}_{\bar{\sigma}, \bar{s}}$ of (1.4) with $\left(\omega_{1}, \omega_{2}\right)=(n / m, 1+\varepsilon)$ satisfying

$$
\left.\left.|u(\varepsilon, \varphi)-| \varepsilon\right|^{\frac{1}{2(d-1)}} \bar{q}_{\varepsilon}(\varphi)\right|_{\bar{\sigma}, \bar{s}} \leq \bar{C} \frac{m^{2}|\varepsilon|}{\gamma \omega_{1}^{3}}|\varepsilon|^{\frac{1}{2(d-1)}}
$$

for an appropriate function $\bar{q}_{\varepsilon} \in \mathcal{H}_{\sigma, s} \backslash\{0\}$ of the form $\bar{q}_{\varepsilon}(\varphi)=\bar{q}_{+}\left(\varphi_{2}\right)+\bar{q}_{-}\left(2 m \varphi_{1}-\right.$ $\left.n \varphi_{2}\right)$.

As a consequence, equation (1.1) admits the quasi-periodic solution $v(\varepsilon, t, x):=$ $u\left(\varepsilon, \omega_{1} t, x+\omega_{2} t\right)$ with two frequencies $\left(\omega_{1}, \omega_{2}\right)=(n / m, 1+\varepsilon)$ and the map $t \rightarrow v(\varepsilon, t, \cdot) \in$ $H^{\bar{\sigma}}(\mathbb{T})$ has the form ${ }^{4}$

$$
\left.\left.|v(\varepsilon, t, x)-| \varepsilon\right|^{\frac{1}{2(d-1)}}\left[\bar{q}_{+}(x+(1+\varepsilon) t)+\bar{q}_{-}((1-\varepsilon) n t-n x)\right]\right|_{H^{\bar{\sigma}}(\mathbb{T})}=O\left(\frac{m^{2}}{\gamma \omega_{1}^{3}}|\varepsilon|^{\frac{2 d-1}{2(d-1)}}\right) .
$$

At the first order the quasi-periodic solution $v(\varepsilon, t, x)$ of equation (1.1) is the superposition of two waves traveling in opposite directions (in general, both components $q_{+}$, $q_{-}$are non trivial).

The bifurcation of quasi-periodic solutions looks quite different if $\omega_{1}$ is irrational.

Theorem B. Let $\omega_{1} \in \mathbb{R} \backslash \mathbb{Q}$. Assume that $f$ satisfies assumption $(\mathbf{H}), \int_{0}^{2 \pi} a_{2 d-1}\left(\varphi_{1}\right) d \varphi_{1}$ $\neq 0$ and $f\left(\varphi_{1}, u\right) \in H^{s}(\mathbb{T}), s \geq 1$, for all $u$.

\footnotetext{
${ }^{2}$ Given $z \in \mathbb{C}, z^{*}$ denotes its complex conjugate.

${ }^{3}$ The proof that $\mathcal{B}_{\gamma} \cap\left(0, \varepsilon_{0}\right)$ and $\mathcal{B}_{\gamma} \cap\left(-\varepsilon_{0}, 0\right)$ are both uncountable $\forall \varepsilon_{0}>0$ is like in BP.

${ }^{4}$ We denote $H^{\sigma}(\mathbb{T}):=\left\{u(\varphi)=\sum_{l \in \mathbb{Z}} \hat{u}_{l} e^{\mathrm{i} l \varphi}: \hat{u}_{l}^{*}=\hat{u}_{-l},|u|_{H^{\sigma}(\mathbb{T})}:=\sum_{l \in \mathbb{Z}}\left|\hat{u}_{l}\right| e^{\sigma|l|}<+\infty\right\}$.
} 
Let $\mathcal{C}_{\gamma} \subset D \equiv\left(-\varepsilon_{0}, \varepsilon_{0}\right) \times(1,2)$ be the uncountable zero-measure Cantor set ${ }^{5}$

$$
\mathcal{C}_{\gamma}:=\left\{\begin{aligned}
\left(\varepsilon, \omega_{1}\right) \in D: & \omega_{1} \notin \mathbb{Q}, \quad \frac{\omega_{1}}{\omega_{2}} \notin \mathbb{Q}, \quad\left|\omega_{1} l_{1}+\varepsilon l_{2}\right|>\frac{\gamma}{\left|l_{1}\right|+\left|l_{2}\right|}, \\
& \left|\omega_{1} l_{1}+(2+\varepsilon) l_{2}\right|>\frac{\gamma}{\left|l_{1}\right|+\left|l_{2}\right|}, \forall l_{1}, l_{2} \in \mathbb{Z} \backslash\{0\}
\end{aligned}\right\} .
$$

Fix any $0<\bar{s}<s-1 / 2$. There exist positive constants $\bar{\varepsilon}, \bar{C}, \bar{\sigma}>0$, such that, $\forall\left(\varepsilon, \omega_{1}\right) \in \mathcal{C}_{\gamma}$ with $|\varepsilon| \gamma^{-1}<\bar{\varepsilon}$ and $\varepsilon \int_{0}^{2 \pi} a_{2 d-1}\left(\varphi_{1}\right) d \varphi_{1}>0$, there exists a nontrivial solution $u(\varepsilon, \varphi) \in \mathcal{H}_{\bar{\sigma}, \bar{s}}$ of equation (1.4) with $\left(\omega_{1}, \omega_{2}\right)=\left(\omega_{1}, 1+\varepsilon\right)$ satisfying

$$
\left.\left.|u(\varepsilon, \varphi)-| \varepsilon\right|^{\frac{1}{2(d-1)}} \bar{q}_{\varepsilon}\left(\varphi_{2}\right)\right|_{\bar{\sigma}, \bar{s}} \leq \bar{C} \frac{|\varepsilon|}{\gamma}|\varepsilon|^{\frac{1}{2(d-1)}}
$$

for some function $\bar{q}_{\varepsilon}\left(\varphi_{2}\right) \in H^{\bar{\sigma}}(\mathbb{T}) \backslash\{0\}$.

As a consequence, equation (1.1) admits the non-trivial quasi-periodic solution $v(\varepsilon, t, x):=$ $u\left(\varepsilon, \omega_{1} t, x+\omega_{2} t\right)$ with two frequencies $\left(\omega_{1}, \omega_{2}\right)=\left(\omega_{1}, 1+\varepsilon\right)$ and the map $t \rightarrow v(\varepsilon, t, \cdot) \in$ $H^{\bar{\sigma}}(\mathbb{T})$ has the form

$$
\left.\left.|v(\varepsilon, t, x)-| \varepsilon\right|^{\frac{1}{2(d-1)}} \bar{q}_{\varepsilon}(x+(1+\varepsilon) t)\right|_{H^{\bar{\sigma}}(\mathbb{T})}=O\left(\gamma^{-1}|\varepsilon|^{\frac{2 d-1}{2(d-1)}}\right) .
$$

Remark 1. Imposing in the definition of $\mathcal{C}_{\gamma}$ the condition $\omega_{1} / \omega_{2}=\omega_{1} /(1+\varepsilon) \in \mathbb{Q}$ we obtain, by Theorem $B$ the existence of periodic solutions of equation (1.1). They are reminiscent, in this completely resonant context, of the Birkhoff-Lewis periodic orbits with large minimal period accumulating at the origin, see [BaB], BBV].

Remark 2. (Non existence) In Theorem B, existence of quasi-periodic solutions could follow by other hypotheses on $f$, see remark [3. However the hypothesis that the leading term in the nonlinearity $f$ is an odd power of $u$ is not of purely technical nature. If $f\left(\varphi_{1}, u\right)=a\left(\varphi_{1}\right) u^{D}$ with $D$ even and $\int_{0}^{2 \pi} a\left(\varphi_{1}\right) d \varphi_{1} \neq 0$, then, $\forall R>0$ there exists $\varepsilon_{0}>0$ such that $\forall \sigma \geq 0, \bar{s}>s-1 / 2, \forall\left(\varepsilon, \omega_{1}\right) \in \mathcal{C}_{\gamma}$ with $|\varepsilon|<\varepsilon_{0}$, equation (1.4) does not possess solutions $u \in \mathcal{H}_{\sigma, \bar{s}}$ in the ball $|u|_{\sigma, \bar{s}} \leq R|\varepsilon|^{1 /(D-1)}$, see Proposition 3 .

To prove Theorems A-B, instead of looking for solutions of equation (1.4) in a shrinking neighborhood of 0 , it is a convenient devise to perform the rescaling

$$
u \rightarrow \delta u \quad \text { with } \quad \delta:=|\varepsilon|^{1 / 2(d-1)}
$$

enhancing the relation between the amplitude $\delta$ and the frequency $\omega_{2}=1+\varepsilon$. We obtain the equation

$$
\mathcal{L}_{\varepsilon} u+\varepsilon f\left(\varphi_{1}, u, \delta\right)=0
$$

where, see (1.3),

$$
\begin{aligned}
\mathcal{L}_{\varepsilon} & :=\left[\omega_{1} \partial_{\varphi_{1}}+\varepsilon \partial_{\varphi_{2}}\right] \circ\left[\omega_{1} \partial_{\varphi_{1}}+(2+\varepsilon) \partial_{\varphi_{2}}\right] \\
& =\left[\omega_{1}^{2} \partial_{\varphi_{1}}^{2}+2 \omega_{1} \partial_{\varphi_{1}} \partial_{\varphi_{2}}\right]+\varepsilon\left[(2+\varepsilon) \partial_{\varphi_{2}}^{2}+2 \omega_{1} \partial_{\varphi_{1}} \partial_{\varphi_{2}}\right]
\end{aligned}
$$

\footnotetext{
${ }^{5}$ See Lemma 3.1
} 
and

$$
f\left(\varphi_{1}, u, \delta\right):=\operatorname{sign}(\varepsilon) \frac{f\left(\varphi_{1}, \delta u\right)}{\delta^{2(d-1)}}=\operatorname{sign}(\varepsilon)\left(a_{2 d-1}\left(\varphi_{1}\right) u^{2 d-1}+\delta a_{2 d}\left(\varphi_{1}\right) u^{2 d}+\ldots\right)
$$

and $\operatorname{sign}(\varepsilon):=1$ if $\omega_{2}>1$ and $\operatorname{sign}(\varepsilon):=-1$ if $\omega_{2}<1$.

To find solutions of equation (1.8) we shall apply the Lyapunov-Schmidt decomposition method which leads to solve separately a "range equation" and a "bifurcation equation".

In order to solve the range equation (avoiding small divisor problems) we restrict $\varepsilon$ to the uncountable zero-measure set $\mathcal{B}_{\gamma}$ for Theorem A, resp. $\left(\varepsilon, \omega_{1}\right) \in \mathcal{C}_{\gamma}$ for Theorem $\mathrm{B}$, and we apply the Contraction Mapping Theorem; similar non-resonance conditions have been employed e.g. in [LS], [BP], [BB1]- $\mathrm{BB} 2,[\mathrm{Mc},[\mathrm{P} 1]$.

To solve the infinite dimensional bifurcation equation we proceed in different ways in case A) and case B).

As already said, in case A) we follow the variational approach of [BB1, [BB2] noting that the bifurcation equation is the Euler-Lagrange equation of a "reduced action functional" which turns out to have the geometry of the infinite dimensional linking theorem of Benci-Rabinowitz [BR. However we can not directly apply the linking theorem because the reduced action functional is defined only in a ball centered at the origin (where the range equation is solved). Moreover the infinite dimensional linking theorem of $\mathrm{BR}$. requires the compactness of the gradient of the functional, property which is not preserved by extending the functional in the whole infinite dimensional space.

In order to overcome these difficulties we perform a further finite dimensional reduction of Galerkin type inspired to [BB3] on a subspace of dimension $N$, with $N$ large but independent of $\varepsilon$, see the equations (2.3)-(2.4)-(2.5).

We shall have to solve the (2.4)-(2.5) equations in a sufficiently large domain of $q_{1}$ (Lemma 2.3), consistent with the $|\cdot|_{H^{1}}$ bounds on the solution $q_{1}$ of the bifurcation equation that can be obtained by the variational arguments, see Lemma 2.6.

Another advantage of this method is that allows to prove the analiticity of the solution $u$ in the variable $\varphi_{2}$.

In case B) the bifurcation equation could be solved through variational methods as in case A). However there is a simpler technique available. The bifurcation equation reduces, in the limit $\varepsilon \rightarrow 0$, to a super-quadratic Hamiltonian system with one degree of freedom. We prove existence of a non-degenerate solution by phase-space analysis. Therefore it can be continued by the Implicit function Theorem to a solution of the complete bifurcation equation for $\varepsilon$ small.

The paper is organized as follows. For simplicity of exposition we prove first Theorem A in the case $\omega_{1}=1$. We deal with the general case $\omega_{1}=\frac{n}{m} \in \mathbb{Q}$ at the end of section [2. In section 3 we prove Theorem B.

Acknowledgments: The authors thank Luca Biasco and Philippe Bolle for useful comments. Part of this paper was written when the second author was at SISSA. 


\section{Case A: $\omega_{1} \in \mathbb{Q}$}

Equation (1.8) becomes, for $\omega_{1}=1$

$$
\mathcal{L}_{\varepsilon} u+\varepsilon f\left(\varphi_{1}, u, \delta\right)=0
$$

where

$$
\begin{aligned}
\mathcal{L}_{\varepsilon} & :=\left[\partial_{\varphi_{1}}+\varepsilon \partial_{\varphi_{2}}\right] \circ\left[\partial_{\varphi_{1}}+(2+\varepsilon) \partial_{\varphi_{2}}\right] \\
& =\left[\partial_{\varphi_{1}}^{2}+2 \partial_{\varphi_{1}} \partial_{\varphi_{2}}\right]+\varepsilon\left[(2+\varepsilon) \partial_{\varphi_{2}}^{2}+2 \partial_{\varphi_{1}} \partial_{\varphi_{2}}\right] \equiv L_{0}+\varepsilon L_{1} .
\end{aligned}
$$

To fix notations we shall prove Theorem A in the case $a_{2 d-1}\left(\varphi_{1}\right)>0$ and $\varepsilon>0$, i.e. $\operatorname{sign}(\varepsilon)>0$.

By the assumption $(\mathbf{H})$ on the nonlinearity $f$ and by the Banach algebra property of $\mathcal{H}_{\sigma, s}$ the Nemitskii operator

$$
u \rightarrow f\left(\varphi_{1}, u, \delta\right) \in C^{\infty}\left(B_{\rho}, \mathcal{H}_{\sigma, s}\right), \quad 0<s<\frac{1}{2}
$$

where $B_{\rho}$ is the ball of radius $\rho \delta^{-1}$ in $\mathcal{H}_{\sigma, s}$ and $\rho$ is connected to the analiticity radius $r$ of $f$ (note that since $a_{k}\left(\varphi_{1}\right) \in H^{1}(\mathbb{T})$ then $a_{k}(\cdot) \in \mathcal{H}_{\sigma, s}, \forall \sigma>0,0<s<1 / 2$ ).

Equation (2.1) is the Euler-Lagrange equation of the Lagrangian action functional $\Psi_{\varepsilon} \in C^{1}\left(\mathcal{H}_{\sigma, s}, \mathbb{R}\right)$ defined by

$$
\begin{aligned}
\Psi_{\varepsilon}(u) & :=\int_{\mathbb{T}^{2}} \frac{1}{2}\left(\partial_{\varphi_{1}} u\right)^{2}+\left(\partial_{\varphi_{1}} u\right)\left(\partial_{\varphi_{2}} u\right)+\frac{\varepsilon(2+\varepsilon)}{2}\left(\partial_{\varphi_{2}} u\right)^{2}+\varepsilon\left(\partial_{\varphi_{1}} u\right)\left(\partial_{\varphi_{2}} u\right)-\varepsilon F\left(\varphi_{1}, u, \delta\right) \\
& \equiv \Psi_{0}(u)+\varepsilon \Gamma(u, \delta)
\end{aligned}
$$

where $F\left(\varphi_{1}, u, \delta\right):=\int_{0}^{u} f\left(\varphi_{1}, \xi, \delta\right) d \xi$ and

$$
\begin{aligned}
\Psi_{0}(u) & :=\int_{\mathbb{T}^{2}} \frac{1}{2}\left(\partial_{\varphi_{1}} u\right)^{2}+\left(\partial_{\varphi_{1}} u\right)\left(\partial_{\varphi_{2}} u\right) \\
\Gamma(u, \delta) & :=\int_{\mathbb{T}^{2}} \frac{(2+\varepsilon)}{2}\left(\partial_{\varphi_{2}} u\right)^{2}+\left(\partial_{\varphi_{1}} u\right)\left(\partial_{\varphi_{2}} u\right)-F\left(\varphi_{1}, u, \delta\right) .
\end{aligned}
$$

To find critical points of $\Psi_{\varepsilon}$ we perform a variational Lyapunov-Schmidt reduction inspired to $[\mathrm{BB} 1]-\mathrm{BB} 2$, see also $[\mathrm{AB}]$.

2.1. The Variational Lyapunov-Schmidt Reduction. The unperturbed functional $\Psi_{0}: \mathcal{H}_{\sigma, s} \rightarrow \mathbb{R}$ possesses an infinite dimensional linear space $Q$ of critical points which are the solutions $q$ of the equation

$$
L_{0} q=\partial_{\varphi_{1}}\left(\partial_{\varphi_{1}}+2 \partial_{\varphi_{2}}\right) q=0
$$

The space $Q$ can be written as

$$
Q=\left\{q=\sum_{l \in \mathbb{Z}^{2}} \hat{q}_{l} e^{\mathrm{i} l \cdot \varphi} \in \mathcal{H}_{\sigma, s} \mid \hat{q}_{l}=0 \quad \text { for } \quad l_{1}\left(l_{1}+2 l_{2}\right) \neq 0\right\}
$$

In view of the variational argument that we shall use to solve the bifurcation equation we split $Q$ as

$$
Q=Q_{+} \oplus Q_{0} \oplus Q_{-}
$$


where $^{6}$

$$
\begin{aligned}
Q_{+} & :=\left\{q \in Q: \hat{q}_{l}=0 \text { for } l \notin \Lambda_{+}\right\}=\left\{q_{+}:=q_{+}\left(\varphi_{2}\right) \in H_{0}^{\sigma}(\mathbb{T})\right\} \\
Q_{0} & :=\left\{q_{0} \in \mathbb{R}\right\} \\
Q_{-} & :=\left\{q \in Q: \hat{q}_{l}=0 \text { for } l \notin \Lambda_{-}\right\}=\left\{q_{-}:=q_{-}\left(2 \varphi_{1}-\varphi_{2}\right), q_{-}(\cdot) \in H_{0}^{\sigma, s}(\mathbb{T})\right\}
\end{aligned}
$$

and

$$
\Lambda_{+}:=\left\{l \in \mathbb{Z}^{2}: l_{1}=0, l \neq 0\right\}, \quad \Lambda_{-}:=\left\{l \in \mathbb{Z}^{2}: l_{1}+2 l_{2}=0, l \neq 0\right\} .
$$

We shall also use in $Q$ the norm

$$
|q|_{H^{1}}^{2}=\left|q_{+}\right|_{H^{1}(\mathbb{T})}^{2}+q_{0}^{2}+\left|q_{-}\right|_{H^{1}(\mathbb{T})}^{2} \sim \sum_{l \in \Lambda_{-} \cup\{0\} \cup \Lambda_{+}} \hat{q}_{l}^{2}\left(|l|^{2}+1\right) .
$$

We decompose the space $\mathcal{H}_{\sigma, s}=Q \oplus P$ where

$$
P:=\left\{p=\sum_{l \in \mathbb{Z}^{2}} \hat{p}_{l} e^{\mathrm{i} l \cdot \varphi} \in \mathcal{H}_{\sigma, s} \mid \hat{p}_{l}=0 \quad \text { for } \quad l_{1}\left(2 l_{2}+l_{1}\right)=0\right\} .
$$

Projecting equation (2.1) onto the closed subspaces $Q$ and $P$, setting $u=q+p \in \mathcal{H}_{\sigma, s}$ with $q \in Q$ and $p \in P$, we obtain

$$
\left\{\begin{array}{l}
L_{1}[q]+\Pi_{Q} f\left(\varphi_{1}, q+p, \delta\right)=0 \\
\mathcal{L}_{\varepsilon}[p]+\varepsilon \Pi_{P} f\left(\varphi_{1}, q+p, \delta\right)=0
\end{array}\right.
$$

where $\Pi_{Q}: \mathcal{H}_{\sigma, s} \rightarrow Q, \Pi_{P}: \mathcal{H}_{\sigma, s} \rightarrow P$ are the projectors respectively onto $Q$ and $P$.

In order to prove analiticity of the solutions and to highlight the compactness of the problem we perform a finite dimensional Lyapunov-Schmidt reduction, introducing the decomposition

where

$$
Q=Q_{1} \oplus Q_{2}
$$

$Q_{1}:=Q_{1}(N):=\left\{q=\sum_{|l| \leq N} \hat{q}_{l} e^{\mathrm{i} l \cdot \varphi} \in Q\right\}, Q_{2}:=Q_{2}(N):=\left\{q=\sum_{|l|>N} \hat{q}_{l} e^{\mathrm{i} l \cdot \varphi} \in Q\right\}$.

Setting $q=q_{1}+q_{2}$ with $q_{1} \in Q_{1}$ and $q_{2} \in Q_{2}$, we finally get

$$
\begin{aligned}
L_{1}\left[q_{1}\right]+\Pi_{Q_{1}}\left[f\left(\varphi_{1}, q_{1}+q_{2}+p, \delta\right)\right]=0 & \Longleftrightarrow d \Psi_{\varepsilon}(u)[h]=0 \quad \forall h \in Q_{1} \quad\left(Q_{1}\right) \\
L_{1}\left[q_{2}\right]+\Pi_{Q_{2}}\left[f\left(\varphi_{1}, q_{1}+q_{2}+p, \delta\right)\right]=0 & \Longleftrightarrow d \Psi_{\varepsilon}(u)[h]=0 \quad \forall h \in Q_{2} \quad\left(Q_{2}\right) \\
\mathcal{L}_{\varepsilon}[p]+\varepsilon \Pi_{P}\left[f\left(\varphi_{1}, q_{1}+q_{2}+p, \delta\right)\right]=0 & \Longleftrightarrow d \Psi_{\varepsilon}(u)[h]=0 \quad \forall h \in P \quad(P)
\end{aligned}
$$

where $\Pi_{Q_{i}}: \mathcal{H}_{\sigma, s} \rightarrow Q_{i}$ are the projectors onto $Q_{i}(i=1,2)$.

We shall solve first the $\left(Q_{2}\right)$ - $(P)$-equations for all $\left|q_{1}\right|_{H^{1}} \leq 2 R$, provided $\varepsilon$ belongs to a suitable Cantor-like set, $|\varepsilon| \leq \varepsilon_{0}(R)$ is sufficiently small and $N \geq N_{0}(R)$ is large enough (see Lemma 2.3).

\footnotetext{
${ }^{6} H_{0}^{\sigma}(\mathbb{T})$ denotes the functions of $H^{\sigma}(\mathbb{T})$ with zero average. $H^{\sigma, s}(\mathbb{T}):=\left\{u(\varphi)=\sum_{l \in \mathbb{Z}} \hat{u}_{l} e^{\mathrm{i} l \varphi}\right.$ : $\left.\hat{u}_{l}^{*}=\hat{u}_{-l},|u|_{H^{\sigma, s}(\mathbb{T})}:=\sum_{l \in \mathbb{Z}}\left|\hat{u}_{l}\right| e^{\sigma|l|}[l]^{s}<+\infty\right\}$ and $H_{0}^{\sigma, s}(\mathbb{T})$ its functions with zero average.
} 
Next we shall solve the $\left(Q_{1}\right)$-equation by means of a variational linking argument, see subsection 2.4 .

2.2. The $\left(Q_{2}\right)$ - $(P)$-equations. We first prove that $\mathcal{L}_{\varepsilon}$ restricted to $P$ has a bounded inverse when $\varepsilon$ belongs to the uncountable zero measure set

$$
\mathcal{B}_{\gamma}:=\left\{\varepsilon \in\left(-\varepsilon_{0}, \varepsilon_{0}\right):\left|l_{1}+\varepsilon l_{2}\right|>\frac{\gamma}{\left|l_{2}\right|}, \forall l_{1}, l_{2} \in \mathbb{Z} \backslash\{0\}\right\}
$$

where $0<\gamma<1 / 6$. $\mathcal{B}_{\gamma}$ accumulates at 0 both from the right and from the left, see $\mathrm{BP}$.

The operator $\mathcal{L}_{\varepsilon}$ is diagonal in the Fourier basis $\left\{e^{\mathrm{i} l \cdot \varphi}, l \in \mathbb{Z}^{2}\right\}$ with eigenvalues $D_{l}:=\left(l_{1}+\varepsilon l_{2}\right)\left(l_{1}+(2+\varepsilon) l_{2}\right)$.

Lemma 2.1. For $\varepsilon \in \mathcal{B}_{\gamma}$ the eigenvalues $D_{l}$ of $\mathcal{L}_{\varepsilon}$ restricted to $P$, satisfy

$$
\left|D_{l}\right|=\left|l_{1}+\varepsilon l_{2}\right|\left|\left(l_{1}+2 l_{2}\right)+\varepsilon l_{2}\right|>\gamma \quad \forall l_{1} \neq 0, l_{1}+2 l_{2} \neq 0 .
$$

As a consequence the operator $\mathcal{L}_{\varepsilon}: P \rightarrow P$ has a bounded inverse $\mathcal{L}_{\varepsilon}^{-1}$ satisfying

$$
\left|\mathcal{L}_{\varepsilon}^{-1}[h]\right|_{\sigma, s} \leq \frac{|h|_{\sigma, s}}{\gamma}, \quad \forall h \in P
$$

Proof. Denoting by $[x]$ the nearest integer close to $x$ and $\{x\}=x-[x]$, we have that $D_{l}>1$ if both $l_{1} \neq-\left[\varepsilon l_{2}\right]$ and $l_{1}+2 l_{2} \neq-\left[\varepsilon l_{2}\right]$. If $l_{1}=-\left[\varepsilon l_{2}\right]$ then

$$
\left|D_{l}\right| \geq \frac{\gamma}{\left|l_{2}\right|}\left(\left|2 l_{2}\right|-\left\{\varepsilon l_{2}\right\}\right) \geq \gamma
$$

In the same way if $l_{1}+2 l_{2}=-\left[\varepsilon l_{2}\right]$ we have $\left|D_{l}\right| \geq \frac{\gamma}{\left|l_{2}\right|}\left(\left|2 l_{2}\right|-\left\{\varepsilon l_{2}\right\}\right) \geq \gamma$.

Lemma 2.2. The operator $L_{1}: Q_{2} \rightarrow Q_{2}$ has bounded inverse $L_{1}^{-1}$ which satisfies

$$
\left|L_{1}^{-1}[h]\right|_{\sigma, s} \leq \frac{|h|_{\sigma, s}}{N^{2}}
$$

Proof. $L_{1}$ is diagonal in the Fourier basis of $Q: e^{\mathrm{il} \cdot \varphi}$ with $l \in \Lambda_{+} \cup\{0\} \cup \Lambda_{-}$(recall (2.2) ) with eigenvalues

$$
d_{l}=(2+\varepsilon) l_{2}^{2} \quad \text { if } l_{1}=0 \text { and } \quad d_{l}=(-2+\varepsilon) l_{2}^{2} \text { if } l_{1}+2 l_{2}=0 .
$$

The eigenvalues of $L_{1}$ restricted to $Q_{2}(N)$ verify $\left|d_{l}\right| \geq(2-\varepsilon) N^{2}$ and (2.7) holds.

Fixed points of the nonlinear operator $\mathcal{G}: Q_{2} \oplus P \rightarrow Q_{2} \oplus P$ defined by

$$
\mathcal{G}\left(q_{2}, p ; q_{1}\right):=\left(-L_{1}^{-1} \Pi_{Q_{2}} f\left(\varphi_{1}, q_{1}+q_{2}+p, \delta\right),-\varepsilon \mathcal{L}_{\varepsilon}^{-1} \Pi_{P} f\left(\varphi_{1}, q_{1}+q_{2}+p, \delta\right)\right)
$$

are solutions of the $\left(Q_{2}\right)-(P)$-equations.

Using the Contraction Mapping Theorem we can prove: 
Lemma 2.3. (Solution of the $\left(Q_{2}\right)-(P)$ equations) $\forall R>0$ there exist an integer $N_{0}(R) \in \mathbb{N}^{+}$and positive constants $\varepsilon_{0}(R)>0, C_{0}(R)>0$ such that:

$$
\forall\left|q_{1}\right|_{H^{1}} \leq 2 R, \quad \forall \varepsilon \in B_{\gamma},|\varepsilon| \gamma^{-1} \leq \varepsilon_{0}(R), \quad \forall N \geq N_{0}(R): 0 \leq \sigma N \leq 1,
$$

there exists a unique solution $\left(q_{2}\left(q_{1}\right), p\left(q_{1}\right)\right):=\left(q_{2}\left(\varepsilon, N, q_{1}\right), p\left(\varepsilon, N, q_{1}\right)\right) \in Q_{2} \oplus P$ of the $\left(Q_{2}\right)-(P)$ equations satisfying

$$
\left|q_{2}\left(\varepsilon, N, q_{1}\right)\right|_{\sigma, s} \leq \frac{C_{0}(R)}{N^{2}}, \quad\left|p\left(\varepsilon, N, q_{1}\right)\right|_{\sigma, s} \leq C_{0}(R)|\varepsilon| \gamma^{-1}
$$

Moreover the map $q_{1} \rightarrow\left(q_{2}\left(q_{1}\right), p\left(q_{1}\right)\right)$ is in $C^{1}\left(B_{2 R}, Q_{2} \oplus P\right)$ and

$$
\left|p^{\prime}\left(q_{1}\right)[h]\right|_{\sigma, s} \leq C_{0}(R)|\varepsilon| \gamma^{-1}|h|_{H^{1}}, \quad\left|q_{2}^{\prime}\left(q_{1}\right)[h]\right|_{\sigma, s} \leq \frac{C_{0}(R)}{N^{2}}|h|_{H^{1}} \quad \forall h \in Q_{1} .
$$

Proof. In the Appendix.

2.3. The $\left(Q_{1}\right)$-equation. Once the $\left(Q_{2}\right)$ - $(P)$-equations have been solved by $\left(q_{2}\left(q_{1}\right)\right.$, $\left.p\left(q_{1}\right)\right) \in Q_{2} \oplus P$ there remains the finite dimensional $\left(Q_{1}\right)$-equation

$$
L_{1}\left[q_{1}\right]+\Pi_{Q_{1}} f\left(\varphi_{1}, q_{1}+q_{2}\left(q_{1}\right)+p\left(q_{1}\right), \delta\right)=0 .
$$

The geometric interpretation of the construction of $\left(q_{2}\left(q_{1}\right), p\left(q_{1}\right)\right)$ is that on the finite dimensional sub-manifold $Z \equiv\left\{q_{1}+q_{2}\left(q_{1}\right)+p\left(q_{1}\right):\left|q_{1}\right|<2 R\right\}$, diffeomorphic to the ball

$$
B_{2 R}:=\left\{q_{1} \in Q_{1}:\left|q_{1}\right|_{H^{1}}<2 R\right\},
$$

the partial derivatives of the action functional $\Psi_{\varepsilon}$ with respect to the variables $\left(q_{2}, p\right)$ vanish. We claim that at a critical point of $\Psi_{\varepsilon}$ restricted to $Z$, also the partial derivative of $\Psi_{\varepsilon}$ w.r.t. the variable $q_{1}$ vanishes and therefore that such point is critical also for the non-restricted functional $\Psi_{\varepsilon}: \mathcal{H}_{\sigma, s} \rightarrow \mathbb{R}$.

Actually the bifurcation equation (2.12) is the Euler-Lagrange equation of the reduced Lagrangian action functional

$$
\Phi_{\varepsilon, N}: B_{2 R} \subset Q_{1} \rightarrow \mathbb{R}, \quad \Phi_{\varepsilon, N}\left(q_{1}\right):=\Psi_{\varepsilon}\left(q_{1}+q_{2}\left(q_{1}\right)+p\left(q_{1}\right)\right) .
$$

Lemma 2.4. $\Phi_{\varepsilon, N} \in \mathcal{C}^{1}\left(B_{2 R}, \mathbb{R}\right)$ and a critical point $q_{1} \in B_{2 R}$ of $\Phi_{\varepsilon, N}$ is a solution of the bifurcation equation (2.12). Moreover $\Phi_{\varepsilon, N}$ can be written as

$$
\Phi_{\varepsilon, N}\left(q_{1}\right)=\text { const }+\varepsilon\left(\Gamma\left(q_{1}\right)+\mathcal{R}_{\varepsilon, N}\left(q_{1}\right)\right)
$$

where

$$
\begin{aligned}
\Gamma\left(q_{1}\right):=\int_{\mathbb{T}^{2}} \frac{(2+\varepsilon)}{2}\left(\partial_{\varphi_{2}} q_{1}\right)^{2}+\left(\partial_{\varphi_{1}} q_{1}\right)\left(\partial_{\varphi_{2}} q_{1}\right)-a_{2 d-1}\left(\varphi_{1}\right) \frac{q_{1}^{2 d}}{2 d} \\
\mathcal{R}_{\varepsilon, N}\left(q_{1}\right):=\int_{\mathbb{T}^{2}} F\left(\varphi_{1}, q_{1}, \delta=0\right)-F\left(\varphi_{1}, q_{1}+q_{2}\left(q_{1}\right)+p\left(q_{1}\right), \delta\right) \\
+\frac{1}{2} f\left(\varphi_{1}, q_{1}+q_{2}\left(q_{1}\right)+p\left(q_{1}\right), \delta\right)\left(q_{2}\left(q_{1}\right)+p\left(q_{1}\right)\right)
\end{aligned}
$$


and, for some positive constant $C_{2}(R) \geq C_{1}(R)$,

$$
\begin{aligned}
\left|\mathcal{R}_{\varepsilon, N}\left(q_{1}\right)\right| & \leq C_{2}(R)\left(\delta+|\varepsilon| \gamma^{-1}+\frac{1}{N^{2}}\right) \\
\left|\mathcal{R}_{\varepsilon, N}^{\prime}\left(q_{1}\right)[h]\right| & \leq C_{2}(R)\left(\delta+|\varepsilon| \gamma^{-1}+\frac{1}{N^{2}}\right)|h|_{H^{1}}, \quad \forall h \in Q_{1} .
\end{aligned}
$$

Proof. In the Appendix.

The problem of finding non-trivial solutions of the $Q_{1}$-equation is reduced to finding non-trivial critical points of the reduced action functional $\Phi_{\varepsilon, N}$ in $B_{2 R}$.

By (2.13), this is equivalent to find critical points of the rescaled functional (still denoted $\Phi_{\varepsilon, N}$ and called the reduced action functional)

$$
\Phi_{\varepsilon, N}\left(q_{1}\right)=\Gamma\left(q_{1}\right)+\mathcal{R}_{\varepsilon, N}\left(q_{1}\right) \equiv\left(\mathcal{A}\left(q_{1}\right)-\int_{\mathbb{T}^{2}} a_{2 d-1}\left(\varphi_{1}\right) \frac{q_{1}^{2 d}}{2 d}\right)+\mathcal{R}_{\varepsilon, N}\left(q_{1}\right)
$$

where the quadratic form

$$
\mathcal{A}(q):=\int_{\mathbb{T}^{2}} \frac{(2+\varepsilon)}{2}\left(\partial_{\varphi_{2}} q\right)^{2}+\left(\partial_{\varphi_{1}} q\right)\left(\partial_{\varphi_{2}} q\right)
$$

is positive definite on $Q_{+}$, negative definite on $Q_{-}$and zero-definite on $Q_{0}$. For $q_{1}=$ $q_{+}+q_{0}+q_{-} \in Q_{1}$,

$$
\mathcal{A}\left(q_{1}\right)=\mathcal{A}\left(q_{+}+q_{0}+q_{-}\right)=\mathcal{A}\left(q_{+}\right)+\mathcal{A}\left(q_{-}\right)=\frac{\alpha_{+}}{2}\left|q_{+}\right|_{H^{1}}^{2}-\frac{\alpha_{-}}{2}\left|q_{-}\right|_{H^{1}}^{2}
$$

for suitable positive constants $\alpha_{+}, \alpha_{-}$, bounded away from 0 by constants independent of $\varepsilon$.

We shall prove the existence of critical points of $\Phi_{\varepsilon, N}$ in $B_{2 R}$ of "linking type".

2.4. Linking critical points of the reduced action functional $\Phi_{\varepsilon, N}$. We can not directly apply the linking Theorem because $\Phi_{\varepsilon, N}$ is defined only in the ball $B_{2 R}$. Therefore our first step is to extend $\Phi_{\varepsilon, N}$ to the whole space $Q_{1}$.

Step 1: Extension of $\Phi_{\varepsilon, N}$. We define the extended action functional $\widetilde{\Phi}_{\varepsilon, N} \in C^{1}\left(Q_{1}, \mathbb{R}\right)$ as

$$
\widetilde{\Phi}_{\varepsilon, N}\left(q_{1}\right):=\Gamma\left(q_{1}\right)+\widetilde{\mathcal{R}}_{\varepsilon, N}\left(q_{1}\right)
$$

where $\widetilde{\mathcal{R}}_{\varepsilon, N}: Q_{1} \rightarrow \mathbb{R}$ is

$$
\widetilde{\mathcal{R}}_{\varepsilon, N}\left(q_{1}\right):=\lambda\left(\frac{\left|q_{1}\right|_{H_{1}}^{2}}{R^{2}}\right) \mathcal{R}_{\varepsilon, N}\left(q_{1}\right)
$$

and $\lambda:[0,+\infty) \rightarrow[0,1]$ is a smooth, non-increasing, cut-off function such that

$$
\left\{\begin{array}{ll}
\lambda(x)=1 & |x| \leq 1 \\
\lambda(x)=0 & |x| \geq 4
\end{array} \quad\left|\lambda^{\prime}(x)\right|<1 .\right.
$$

By definition $\widetilde{\Phi}_{\varepsilon, N} \equiv \Phi_{\varepsilon, N}$ on $B_{R}:=\left\{q_{1} \in Q_{1}:\left|q_{1}\right|_{H^{1}} \leq R\right\}$ and $\widetilde{\Phi}_{\varepsilon, N} \equiv \Gamma$ outside $B_{2 R}$. 
Moreover, by (2.14) -(2.15), there is a constant $C_{3}(R) \geq C_{2}(R)>0$ such that $\forall\left|q_{1}\right|_{H^{1}} \leq 2 R$

$$
\begin{aligned}
\left|\widetilde{\mathcal{R}}_{\varepsilon, N}\left(q_{1}\right)\right| & \leq C_{3}(R)\left(\delta+|\varepsilon| \gamma^{-1}+\frac{1}{N^{2}}\right) \\
\left|\widetilde{\mathcal{R}}_{\varepsilon, N}^{\prime}\left(q_{1}\right)[h]\right| & \leq C_{3}(R)\left(\delta+|\varepsilon| \gamma^{-1}+\frac{1}{N^{2}}\right)|h|_{H^{1}}, \quad \forall h \in Q_{1} .
\end{aligned}
$$

In the sequel we shall always assume

$$
C_{3}(R)\left(\delta+|\varepsilon| \gamma^{-1}+\frac{1}{N^{2}}\right) \leq 1
$$

Step 2: $\widetilde{\Phi}_{\varepsilon, N}$ verifies the geometrical hypotheses of the linking Theorem.

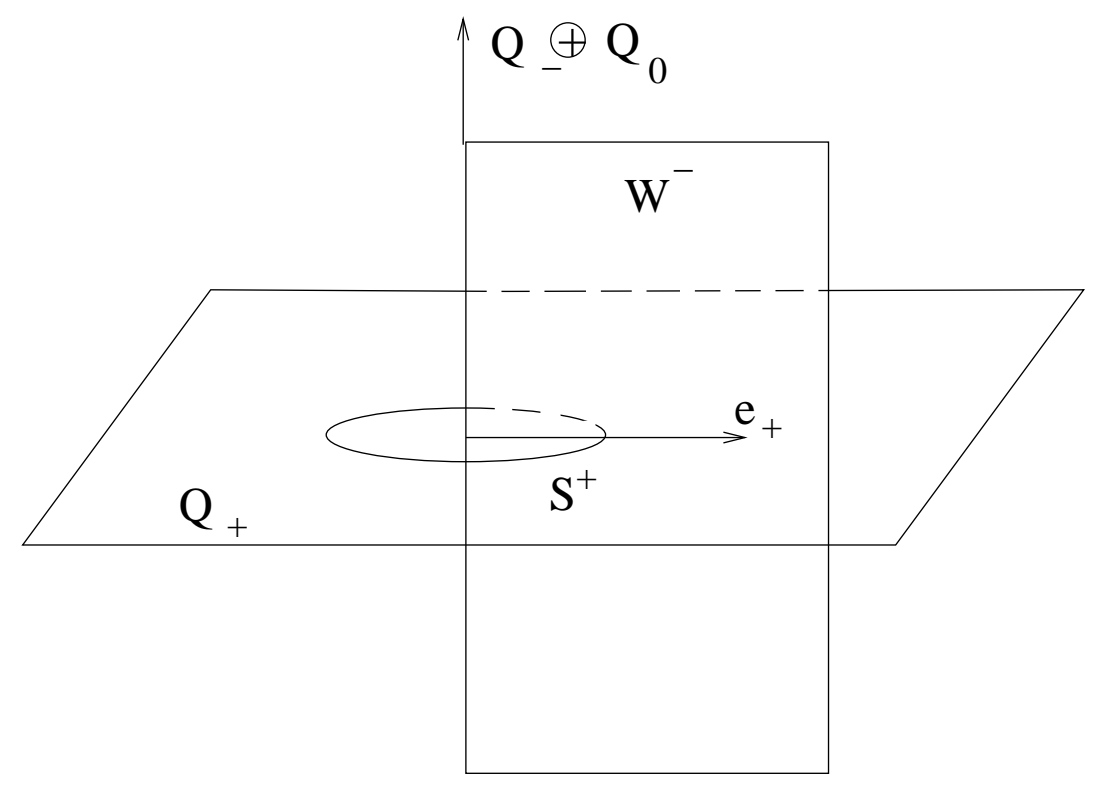

Figure 1 . The cylinder $W^{-}$and the sphere $S^{+}$link.

Lemma 2.5. There exist $\varepsilon-N-\gamma$-independent positive constants $\rho, \omega, r_{1}, r_{2}>\rho$, and $0<\varepsilon_{1}(R) \leq \varepsilon_{0}(R), N_{1}(R) \geq N_{0}(R)$ such that, $\forall|\varepsilon| \gamma^{-1} \leq \varepsilon_{1}(R), \forall N \geq N_{1}(R)$

(i) $\widetilde{\Phi}_{\varepsilon, N}\left(q_{1}\right) \geq \omega>0, \forall q_{1} \in S^{+}:=\left\{q_{1} \in Q_{1} \cap Q_{+}:\left|q_{1}\right|_{H_{1}}=\rho\right\}$,

(ii) $\widetilde{\Phi}_{\varepsilon, N}\left(q_{1}\right) \leq \omega / 2, \forall q_{1} \in \partial W^{-}$where $W^{-}$is the cylinder

$$
W^{-}:=\left\{q_{1}=q_{0}+q_{-}+r e^{+},\left|q_{0}+q_{-}\right|_{H_{1}} \leq r_{1}, q_{-} \in Q_{1} \cap Q_{-}, q_{0} \in \mathbb{R}, r \in\left[0, r_{2}\right]\right\}
$$

and $e_{+}:=\cos \left(\varphi_{2}\right) \in Q_{1} \cap Q_{+}$. Note that $\rho, \omega$ are independent of $R$.

In the following $\kappa_{i}, \kappa_{ \pm}$will denote positive constants independent on $R, N, \varepsilon$ and $\gamma$. 
Proof. (i) $\forall q_{+} \in Q_{1} \cap Q_{+}$with $\left|q_{+}\right|_{H^{1}}=\rho<R$ we have

$$
\begin{aligned}
\widetilde{\Phi}_{\varepsilon, N}\left(q_{+}\right)=\Phi_{\varepsilon, N}\left(q_{+}\right) & =\mathcal{A}\left(q_{+}\right)-\int_{\mathbb{T}^{2}} a_{2 d-1}\left(\varphi_{1}\right) \frac{q_{+}^{2 d}}{2 d}+\mathcal{R}_{\varepsilon, N}\left(q_{+}\right) \\
& \geq \frac{\alpha_{+}}{2} \rho^{2}-\kappa_{1} \rho^{2 d}-\left(\delta+|\varepsilon| \gamma^{-1}+\frac{1}{N^{2}}\right) C_{3}(R) .
\end{aligned}
$$

Now we fix $\rho>0$ small such that $\left(\alpha_{+} \rho^{2} / 2\right)-\kappa_{1} \rho^{2 d} \geq \alpha_{+} \rho^{2} / 4$. Next, for $\left(\delta+|\varepsilon| \gamma^{-1}+\right.$ $\left.N^{-2}\right) C_{3}(R) \leq \alpha_{+} \rho^{2} / 8$ we get by (2.20)

$$
\widetilde{\Phi}_{\varepsilon, N}\left(q_{+}\right) \geq \frac{\alpha_{+}}{8} \rho^{2}=: \omega>0, \quad \forall q_{+} \in Q_{1} \cap Q^{+} \quad \text { with } \quad\left|q_{+}\right|=\rho .
$$

(ii) Let

$$
\begin{aligned}
& B_{1}:=\left\{q_{1}=q_{0}+q_{-}+r_{2} e_{+} \text {with }\left|q_{0}+q_{-}\right|_{H^{1}} \leq r_{1}, q_{-} \in Q_{1} \cap Q_{-}\right\} \subset \partial W^{-} \\
& B_{2}:=\left\{q_{1}=q_{0}+q_{-}+r e_{+} \text {with }\left|q_{0}+q_{-}\right|_{H^{1}}=r_{1}, q_{-} \in Q_{1} \cap Q_{-}, r \in\left[0, r_{2}\right]\right\} \subset \partial W^{-}
\end{aligned}
$$
and choose $r_{1}, r_{2}>2 R$. For $q_{1}=q_{0}+q_{-}+r e_{+} \in B_{1} \cup B_{2}$

$$
\begin{aligned}
\widetilde{\Phi}_{\varepsilon, N}\left(q_{1}\right)=\Gamma\left(q_{1}\right) & =\mathcal{A}\left(q_{1}\right)-\int_{\mathbb{T}^{2}} a_{2 d-1}\left(\varphi_{1}\right)\left(q_{0}+q_{-}+r e_{+}\right)^{2 d} \\
& =-\frac{\alpha_{-}}{2}\left|q_{-}\right|_{H^{1}}^{2}+r^{2} \mathcal{A}\left(e_{+}\right)-\int_{\mathbb{T}^{2}} a_{2 d-1}\left(\varphi_{1}\right) \frac{\left(q_{0}+q_{-}+r e_{+}\right)^{2 d}}{2 d} \\
& \leq-\frac{\alpha_{-}}{2}\left|q_{-}\right|_{H^{1}}^{2}+r^{2} \mathcal{A}\left(e_{+}\right)-\alpha \int_{\mathbb{T}^{2}}\left(q_{0}+q_{-}+r e_{+}\right)^{2 d}
\end{aligned}
$$

because $a_{2 d-1}\left(\varphi_{1}\right) / 2 d \geq \alpha>0$. Now, by Hölder inequality and orthogonality

$$
\begin{aligned}
\int_{\mathbb{T}^{2}}\left(q_{0}+q_{-}+r e_{+}\right)^{2 d} & \geq \kappa_{2}\left(\int_{\mathbb{T}^{2}}\left(q_{0}+q_{-}+r e_{+}\right)^{2}\right)^{d} \\
& =\kappa_{2}\left(\int_{\mathbb{T}^{2}} q_{0}^{2}+q_{-}^{2}+r^{2} e_{+}^{2}\right)^{d} \\
& \geq \kappa_{3}\left(q_{0}^{2}+r^{2}\right)^{d} \geq \kappa_{3}\left(q_{0}^{2 d}+r^{2 d}\right)
\end{aligned}
$$

and by (2.21) we deduce

$$
\widetilde{\Phi}_{\varepsilon, N}\left(q_{0}+q_{-}+r e_{+}\right) \leq\left(\kappa_{+} r^{2}-\kappa_{3} r^{2 d}\right)-\left(\frac{\alpha_{-}}{2}\left|q_{-}\right|_{H^{1}}^{2}+\kappa_{3} q_{0}^{2 d}\right) .
$$

Now we fix $r_{2}$ large such that $\kappa_{+} r_{2}^{2}-\kappa_{3} r_{2}^{2 d} \leq 0$ and therefore

$$
\widetilde{\Phi}_{\varepsilon, N}\left(q_{1}\right) \leq \kappa_{+} r_{2}^{2}-\kappa_{3} r_{2}^{2 d} \leq 0 \quad \forall q_{1} \in B_{1} .
$$

Next, setting $M:=\max _{r \in\left[0, r_{2}\right]}\left(\kappa_{+} r^{2}-\kappa_{3} r^{2 d}\right)$, we fix $r_{1}$ large such that

$$
\frac{\alpha_{-}}{2}\left|q_{-}\right|_{H^{1}}^{2}+\kappa_{3} q_{0}^{2 d} \geq M \quad \forall\left|q_{-}+q_{0}\right|=r_{1}
$$

and therefore

$$
\widetilde{\Phi}_{\varepsilon, N}\left(q_{1}\right) \leq M-\left(\frac{\alpha_{-}}{2}\left|q_{-}\right|_{H^{1}}^{2}+\kappa_{3} q_{0}^{2 d}\right) \leq 0 \quad \forall q_{1} \in B_{2} .
$$


Finally if $q_{1}=q_{-}+q_{0}$ :

$$
\begin{aligned}
\widetilde{\Phi}_{\varepsilon, N}\left(q_{1}\right) & =\mathcal{A}\left(q_{-}\right)-\int_{\mathbb{T}^{2}} a_{2 d-1}\left(\varphi_{1}\right) \frac{q_{1}^{2 d}}{2 d}+\widetilde{\mathcal{R}}_{\varepsilon, N}\left(q_{1}\right) \\
& \leq\left|\widetilde{\mathcal{R}}_{\varepsilon, N}\left(q_{1}\right)\right| \leq C_{3}(R)\left(\delta+|\varepsilon| \gamma^{-1}+N^{-2}\right)
\end{aligned}
$$

and so $\widetilde{\Phi}_{\varepsilon, N}\left(q_{1}\right) \leq \omega / 2$ if $C_{3}(R)\left(\delta+|\varepsilon| \gamma^{-1}+N^{-2}\right) \leq \omega / 2$.

We introduce the minimax class

$$
\mathcal{S}:=\left\{\psi \in C\left(\overline{W^{-}}, Q\right) \mid \psi=\text { Id on } \partial W^{-}\right\} .
$$

The maps of $\mathcal{S}$ have an important intersection property, see e.g. Proposition 5.9 of R3.

\section{Proposition 1. ( $S^{+}$and $W^{-}$link with respect to $\mathcal{S}$.)}

$$
\psi \in \mathcal{S} \quad \Longrightarrow \quad \psi\left(W^{-}\right) \cap S^{+} \neq \emptyset .
$$

Define the minimax linking level

$$
\mathcal{K}_{\varepsilon, N}:=\inf _{\psi \in \mathcal{S}} \max _{q \in W^{-}} \widetilde{\Phi}_{\varepsilon, N}\left(\psi\left(q_{1}\right)\right) .
$$

By the intersection property of Proposition 1 and Lemma 2.5- $(i)$

$$
\max _{q_{1} \in W^{-}} \widetilde{\Phi}_{\varepsilon, N}\left(\psi\left(q_{1}\right)\right) \geq \min _{q_{1} \in S^{+}} \widetilde{\Phi}_{\varepsilon, N}\left(q_{1}\right) \geq \omega>0 \quad \forall \psi \in \mathcal{S}
$$

and therefore

$$
\mathcal{K}_{\varepsilon, N}>\omega>0
$$

Moreover, since Id $\in \mathcal{S}$ and (2.18)

$$
\begin{aligned}
\mathcal{K}_{\varepsilon, N} & \leq \max _{q_{1} \in W^{-}} \widetilde{\Phi}_{\varepsilon, N}\left(q_{1}\right) \leq \max _{q_{1} \in W^{-}}\left(\Gamma\left(q_{1}\right)+\widetilde{\mathcal{R}}_{\varepsilon, N}\left(q_{1}\right)\right) \\
& \leq \max _{q_{1} \in W^{-}}\left(\frac{\alpha_{+}}{2}\left|q_{+}\right|_{H^{1}}^{2}+\frac{\alpha_{-}}{2}\left|q_{-}\right|_{H^{1}}^{2}+\int_{\mathbb{T}^{2}} \kappa q_{1}^{2 d}\right)+1 \leq \mathcal{K}_{\infty}<+\infty
\end{aligned}
$$

where $\mathcal{K}_{\infty}$ is independent of $N, \varepsilon, \gamma$ since the constants $r_{1}, r_{2}$ in the definition of $W^{-}$ are independent of $N, \varepsilon, \gamma$.

We deduce, by the linking theorem the existence of a (Palais-Smale) sequence $\left(q_{j}\right) \in$ $Q_{1}$ at the level $\mathcal{K}_{\varepsilon, N}$, namely

$$
\widetilde{\Phi}_{\varepsilon, N}\left(q_{j}\right) \rightarrow \mathcal{K}_{\varepsilon, N}, \quad \widetilde{\Phi}_{\varepsilon, N}^{\prime}\left(q_{j}\right) \rightarrow 0
$$

Step 3: Existence of a nontrivial critical point. Our final aim is to prove that the Palais-Smale sequence $q_{j}$ converges, up to subsequence, to some non-trivial critical point $\bar{q}_{1} \neq 0$ in some open ball of $Q_{1}$ where $\widetilde{\Phi}_{\varepsilon, N}$ and $\Phi_{\varepsilon, N}$ coincide.

Lemma 2.6. There exists a constant $R_{*}>0$, independent on $R-\varepsilon-N-\gamma$, and functions $0<\varepsilon_{2}(R) \leq \varepsilon_{1}(R), N_{2}(R) \geq N_{1}(R)$ such that for all $|\varepsilon| \gamma^{-1} \leq \varepsilon_{2}(R), N \geq N_{2}(R)$ the functional $\widetilde{\Phi}_{\varepsilon, N}$ possesses a non-trivial critical point $\bar{q}_{1} \in Q_{1}$ with critical value $\widetilde{\Phi}_{\varepsilon, N}\left(\bar{q}_{1}\right)=\mathcal{K}_{\varepsilon, N}$, satisfying $\left|\bar{q}_{1}\right|_{H_{1}} \leq R_{*}$. 
Proof. Writing $\widetilde{\Phi}_{\varepsilon, N}(q)=\Gamma(q)+\widetilde{\mathcal{R}}_{\varepsilon, N}(q)$ we derive, by (2.18) $-(2.19)$

$$
\begin{aligned}
\widetilde{\Phi}_{\varepsilon, N}\left(q_{j}\right)-\frac{1}{2} \widetilde{\Phi}_{\varepsilon, N}^{\prime}\left(q_{j}\right)\left[q_{j}\right] & =\Gamma\left(q_{j}\right)-\frac{1}{2} \Gamma^{\prime}\left(q_{j}\right)\left[q_{j}\right]+\left(\widetilde{\mathcal{R}}_{\varepsilon, N}\left(q_{j}\right)-\frac{1}{2} \widetilde{\mathcal{R}}_{\varepsilon, N}^{\prime}\left(q_{j}\right)\left[q_{j}\right]\right) \\
& =\left(\frac{1}{2}-\frac{1}{2 d}\right) \int_{\mathbb{T}^{2}} a_{2 d-1}\left(\varphi_{1}\right) q_{j}^{2 d}+\left(\widetilde{\mathcal{R}}_{\varepsilon, N}\left(q_{j}\right)-\frac{1}{2} \widetilde{\mathcal{R}}_{\varepsilon, N}^{\prime}\left(q_{j}\right)\left[q_{j}\right]\right) \\
& \geq \alpha\left(\frac{1}{2}-\frac{1}{2 d}\right) \int_{\mathbb{T}^{2}} q_{j}^{2 d}-\left(\delta+|\varepsilon| \gamma^{-1}+N^{-2}\right) C_{3}(R) .
\end{aligned}
$$

Therefore, by (2.23)-(2.24)

$$
\mathcal{K}_{\infty}+1+\left|q_{j}\right|_{H_{1}} \geq \kappa_{1} \int_{\mathbb{T}^{2}} q_{j}^{2 d}:=\kappa_{1}\left|q_{j}\right|_{L^{2 d}}^{2 d} .
$$

We also deduce, by (2.25), Hölder inequality and orthogonality

$$
\begin{aligned}
\mathcal{K}_{\infty}+1+\left|q_{j}\right|_{H_{1}} & \geq \kappa_{2}\left(\int_{\mathbb{T}^{2}}\left(q_{+, j}+q_{0, j}+q_{-, j}\right)^{2}\right)^{d} \\
& =\kappa_{2}\left(\int_{\mathbb{T}^{2}} q_{+, j}^{2}+q_{0, j}^{2}+q_{-, j}^{2}\right)^{d} \geq \kappa_{3}\left(q_{0, j}\right)^{2 d}
\end{aligned}
$$

and therefore

$$
\left|q_{0, j}\right| \leq \kappa_{4}\left(1+\left|q_{j}\right|_{H_{1}}\right)^{1 / 2 d}
$$

By (2.18)-(2.19) and Hölder inequality

$$
\begin{aligned}
\widetilde{\Phi}_{\varepsilon, N}^{\prime}\left(q_{j}\right)\left[q_{+, j}\right] & =\alpha_{+}\left|q_{+, j}\right|_{H_{1}}^{2}-\int_{\mathbb{T}^{2}} a_{2 d-1}\left(\varphi_{1}\right) q_{j}^{2 d-1} q_{+, j}+\widetilde{\mathcal{R}}_{\varepsilon, N}^{\prime}\left(q_{j}\right)\left[q_{+, j}\right] \\
& \geq \alpha_{+}\left|q_{+, j}\right|_{H_{1}}^{2}-\kappa_{5}\left|q_{+, j}\right|_{H_{1}} \int_{\mathbb{T}^{2}}\left|q_{j}\right|^{2 d-1}-\left(\delta+\gamma^{-1}|\varepsilon|+N^{-2}\right) C_{3}(R)\left|q_{+, j}\right|_{H_{1}} \\
(2.27) & \geq \kappa_{6}\left|q_{+, j}\right|_{H_{1}}\left(\left|q_{+, j}\right|_{H_{1}}-\left|q_{j}\right|_{L^{2 d}}^{2 d-1}-1\right) .
\end{aligned}
$$

By (2.27) and (2.25), using that $\widetilde{\Phi}_{\varepsilon, N}^{\prime}\left(q_{j}\right) \rightarrow 0$ and simple inequalities, we conclude

$$
\left|q_{+, j}\right|_{H_{1}} \leq \kappa_{7}\left(1+\left|q_{j}\right|_{H_{1}}^{(2 d-1) / 2 d}\right) \text {. }
$$

Estimating analogously $\widetilde{\Phi}_{\varepsilon, N}^{\prime}\left(q_{j}\right)\left[q_{-, j}\right]$ we derive

$$
\left|q_{-, j}\right|_{H_{1}} \leq \kappa_{8}\left(1+\left|q_{j}\right|_{H_{1}}^{(2 d-1) / 2 d}\right)
$$

and by (2.26) we finally deduce

$$
\left|q_{j}\right|_{H_{1}}=\left|q_{0, j}\right|+\left|q_{+, j}\right|_{H_{1}}+\left|q_{-, j}\right|_{H_{1}} \leq \kappa_{9}\left(1+\left|q_{j}\right|_{H_{1}}^{1 / 2 d}+\left|q_{j}\right|_{H_{1}}^{(2 d-1) / 2 d}\right) .
$$

We conclude that $\left|q_{j}\right|_{H_{1}} \leq R_{*}$ for a suitable positive constant $R_{*}$ independent of $\varepsilon, N$, $R$ and $\gamma$.

Since $Q_{1}$ is finite dimensional $q_{j}$ converges, up to subsequence, to some critical point $\bar{q}_{1}$ of $\widetilde{\Phi}_{\varepsilon, N}$ with $\left|\bar{q}_{1}\right|_{H_{1}} \leq R_{*}$. Finally, since $\widetilde{\Phi}_{\varepsilon, N}\left(\bar{q}_{1}\right)=\mathcal{K}_{\varepsilon, N} \geq \omega>0$ we conclude that $\bar{q}_{1} \neq 0$. 
We are now ready to prove Theorem $\mathrm{A}$ in the case $\omega_{1}=1$.

Proof of Theorem $A$ for $\omega_{1}=1$. Let us fix

$$
\bar{R}:=R_{*}+1 \quad \text { and take } \quad|\varepsilon| \gamma^{-1} \leq \varepsilon_{2}(\bar{R}):=\bar{\varepsilon} .
$$

Set $\bar{N}:=N_{2}(\bar{R}) \geq N_{0}(\bar{R})$.

Applying Lemma 2.3 we obtain, for

$$
0<\sigma \leq \frac{1}{N_{2}(\bar{R})}
$$

a solution $\left(q_{2}\left(q_{1}\right), p\left(q_{1}\right)\right) \in\left(Q_{2}(\bar{N}) \oplus P\right) \cap \mathcal{H}_{\sigma, s}$ of the $\left(Q_{2}\right)$ - $(P)$ equations $\forall\left|q_{1}\right|_{H_{1}} \leq 2 \bar{R}$. By Lemma 2.6 the extended functional $\widetilde{\Phi}_{\varepsilon, N}\left(q_{1}\right)$ possesses a critical point $\bar{q}_{1} \neq 0$ with $\left|\bar{q}_{1}\right|_{H_{1}} \leq R_{*}<\bar{R}$. Since $\widetilde{\Phi}_{\varepsilon, N}\left(q_{1}\right)$ coincides with $\Phi_{\varepsilon, N}\left(q_{1}\right)$ on the ball $B_{\bar{R}}$ we get, by Lemma 2.4, the existence of a nontrivial weak solution $\bar{q}_{1}+q_{2}\left(\bar{q}_{1}\right)+p\left(\bar{q}_{1}\right) \in \mathcal{H}_{\sigma, s}$ of equation (2.1). Finally

$$
u=|\varepsilon|^{1 / 2(d-1)}\left[\bar{q}_{1}+q_{2}\left(\bar{q}_{1}\right)+p\left(\bar{q}_{1}\right)\right] \equiv|\varepsilon|^{1 / 2(d-1)}\left[\bar{q}_{\varepsilon}+p\left(\bar{q}_{1}\right)\right]
$$

solves equation (1.4).

The solution $\bar{q}_{\varepsilon}:=\bar{q}_{1}+q_{2}\left(\bar{q}_{1}\right)$ of the $(Q)$-equation belongs to $Q \cap \mathcal{H}_{\sigma, s+2}$ by the regularizing properties of $L_{1}^{-1}$, see in Lemma 2.2 formula (2.8).

Since $\bar{p}:=p\left(\bar{q}_{1}\right)$ solves

$$
\left(\partial_{\varphi_{1}}^{2}+2(1+\varepsilon) \partial_{\varphi_{1}} \partial_{\varphi_{2}}\right) \bar{p}=-\varepsilon\left[(2+\varepsilon) \partial_{\varphi_{2}}^{2} \bar{p}+\Pi_{P} f\left(\varphi_{1}, u, \delta\right)\right] \in \mathcal{H}_{\sigma^{\prime}, s} \quad \forall 0<\sigma^{\prime}<\sigma
$$

and the eigenvalues of $\partial_{\varphi_{1}}^{2}+2(1+\varepsilon) \partial_{\varphi_{1}} \partial_{\varphi_{2}}$ restricted to $P$ satisfy, for $\varepsilon \in \mathcal{B}_{\gamma}$,

$$
\left|l_{1}\left[\left(l_{1}+2 l_{2}\right)+\varepsilon 2 l_{2}\right]\right| \geq \gamma \frac{\left|l_{1}\right|}{2\left|l_{2}\right|} \quad \forall l_{1}+2 l_{2} \neq 0, l_{2} \neq 0
$$

and we deduce that $\bar{p} \in \mathcal{H}_{\sigma^{\prime \prime}, s+1}$ for all $0<\sigma^{\prime \prime}<\sigma^{\prime}$ and $\left|\partial_{\varphi_{1}} \bar{p}\right|_{\sigma^{\prime \prime}, s}=O\left(|\varepsilon| \gamma^{-1}\right)$. Now, again by (2.28),

$$
\partial_{\varphi_{1}}^{2} \bar{p}=-2(1+\varepsilon) \partial_{\varphi_{2}} \partial_{\varphi_{1}} \bar{p}-\varepsilon\left[(2+\varepsilon) \partial_{\varphi_{2}}^{2} \bar{p}+\Pi_{P} f\left(\varphi_{1}, u, \delta\right)\right] \in \mathcal{H}_{\bar{\sigma}, s} \quad \forall 0<\bar{\sigma}<\sigma^{\prime \prime} .
$$

therefore $\bar{p} \in \mathcal{H}_{\bar{\sigma}, s+2}$ and $|\bar{p}|_{\bar{\sigma}, s+2}=O\left(|\varepsilon| \gamma^{-1}\right)$. (1.5) follows with $\bar{s}:=s+2>2$.

By (1.2), the function $v(\varepsilon, t, x)=u(\varepsilon, t, x+(1+\varepsilon) t)$ is a solution of equation (1.1) with $\omega_{1}=1$. The frequency $\omega_{2}=1+\varepsilon \notin \mathbb{Q}$ since $\varepsilon \in \mathcal{B}_{\gamma}$. To show that $v(\varepsilon, t, x)$ is quasi-periodic it remains to prove that $u$ depends on both the variables $\left(\varphi_{1}, \varphi_{2}\right)$ independently.

We claim that $\bar{q}_{1} \notin Q_{0} \oplus Q_{-}$, i.e. $\bar{q}_{+}\left(\varphi_{2}\right) \in Q_{+} \backslash\{0\}$, and therefore $u$ depends on $\varphi_{2}$. Indeed by Lemma 2.6 we know that $\tilde{\Phi}_{\varepsilon, N}\left(\bar{q}_{1}\right)>\omega>0$ and $\left|\bar{q}_{1}\right|_{H^{1}(\mathbb{T})}<\bar{R}$. On the other hand, by (2.22) in Lemma $2.5 \tilde{\Phi}_{\varepsilon, N}\left(q_{-}+q_{0}\right)<\omega / 2$, for all $\left|q_{-}+q_{0}\right|_{H^{1}} \leq \bar{R}$, so that necessarily $\bar{q}_{1} \notin Q_{0} \oplus Q_{-}$.

We claim that any solution $u$ of (2.1) depending only on $\varphi_{2}$, namely solving

$$
(2+\varepsilon) u^{\prime \prime}\left(\varphi_{2}\right)+f\left(\varphi_{1}, u\left(\varphi_{2}\right), \delta\right)=0,
$$


is $u\left(\varphi_{2}\right) \equiv 0$. Indeed, by definition,

$$
\delta^{2(d-1)} f\left(\varphi_{1}, u, \delta\right)=f\left(\varphi_{1}, \delta u\right)=\sum_{k=2 d-1}^{\infty} a_{k}\left(\varphi_{1}\right)(\delta u)^{k}
$$

(recall $\operatorname{sign}(\varepsilon)=1)$. Consider now a smooth zero mean function $g\left(\varphi_{1}\right)$ such that $\int_{0}^{2 \pi} a_{k}\left(\varphi_{1}\right) g\left(\varphi_{1}\right) \neq 0$ for some $k$ (recall that by assumption $(\mathbf{H})$ some of the $a_{k}\left(\varphi_{2}\right)$ are not constant). By (2.29) we have

$$
(2+\varepsilon) u^{\prime \prime}\left(\varphi_{2}\right) \int_{0}^{2 \pi} g\left(\varphi_{1}\right) d \varphi_{1}+\int_{0}^{2 \pi} f\left(\varphi_{1}, u\left(\varphi_{2}\right), \delta\right) g\left(\varphi_{1}\right) d \varphi_{1}=0
$$

which implies, by the assumption $(\mathbf{H})$ on $f$,

$$
\sum_{k=2 d-1}^{\infty}\left[\delta u\left(\varphi_{2}\right)\right]^{k} \int_{0}^{2 \pi} a_{k}\left(\varphi_{1}\right) g\left(\varphi_{1}\right) d \varphi_{1}=0 .
$$

The function $G(z):=\sum_{k=2 d-1}^{\infty} b_{k} z^{k}$ with $b_{k}:=\int_{0}^{2 \pi} a_{k}\left(\varphi_{1}\right) g\left(\varphi_{1}\right) d \varphi_{1}$ is a nontrivial analytic function. Therefore equation (2.30), i.e. $G\left(\delta u\left(\varphi_{2}\right)\right)=0$, cannot have a sequence of zeros accumulating to 0 . So, for $\delta$ small enough, $u\left(\varphi_{2}\right) \equiv 0$.

Proof of Theorem A for any rational frequency $\omega_{1}=\frac{n}{m} \in \mathbb{Q}$. Consider now equation (1.8) with $\omega_{1}=n / m$ where $n, m$ are coprime integers.

The space $Q$, formed by the solutions of $\partial_{\varphi_{1}}\left(\frac{n}{m} \partial_{\varphi_{1}}+2 \partial_{\varphi_{2}}\right) q=0$ can be written as

$$
Q=\left\{q=\sum_{l \in \mathbb{Z}^{2}} \hat{q}_{l} e^{\mathrm{i} l \cdot \varphi} \in \mathcal{H}_{\sigma, s} \mid \hat{q}_{l}=0 \quad \text { for } \quad l_{1}\left(n l_{1}+2 m l_{2}\right) \neq 0\right\}
$$

and is composed by functions of the form

$$
q(\varphi)=q_{+}\left(\varphi_{2}\right)+q_{-}\left(2 m \varphi_{1}-n \varphi_{2}\right)+q_{0} .
$$

Let $P$ be the supplementary space to $Q$ and perform the Lyapunov-Schmidt decomposition like in (2.3) $-(2.4)-(2.5)$.

For $\varepsilon$ in the Cantor set $\mathcal{B}_{\gamma}$, the eigenvalues

$$
D_{l}=\left(\frac{n}{m} l_{1}+\varepsilon l_{2}\right)\left(\frac{n}{m} l_{1}+2 l_{2}+\varepsilon l_{2}\right)
$$

of the linear operator $\mathcal{L}_{\varepsilon}$ can be bounded, arguing as in Lemma 2.1, by

$$
\left|D_{l}\right|=\frac{\left|\left(n l_{1}+\varepsilon m l_{2}\right)\left(n l_{1}+2 m l_{2}+\varepsilon m l_{2}\right)\right|}{m^{2}}>\frac{\gamma}{m^{2}} \quad \forall l_{1} \neq 0, n l_{1}+2 m l_{2} \neq 0 .
$$

As a consequence

$$
\left|\mathcal{L}_{\varepsilon}^{-1}[h]\right|_{\sigma, s} \leq \frac{m^{2}|h|_{\sigma, s}}{\gamma}, \quad \forall h \in P,
$$

and, in solving the $\left(Q_{2}\right)-(P)$ equations as in Lemma 2.3, we obtain the new restriction

$$
\gamma^{-1}|\varepsilon| \leq \frac{\varepsilon_{0}(R)}{m^{2}}, \quad N \geq N_{0}(R)
$$

and the bound (compare with (2.10) $)\left|p\left(q_{1}\right)\right|_{\sigma, s} \leq C_{0}(R)|\varepsilon| \gamma^{-1} m^{2}$. 
The corresponding reduced action functional has again the form (2.13)-(2.16) with the different quadratic part

$$
\mathcal{A}\left(q_{1}\right)=\mathcal{A}\left(q_{+}+q_{0}+q_{-}\right)=\mathcal{A}\left(q_{+}\right)+\mathcal{A}\left(q_{-}\right)=\frac{\alpha_{+}}{2}\left|q_{+}\right|_{H^{1}}^{2}-n^{2} \frac{\alpha_{-}}{2}\left|q_{-}\right|_{H^{1}}^{2}
$$

and therefore it still possesses a linking critical point $\bar{q}_{1} \in Q_{1}$.

To prove the bound (1.5) note that the eigenvalues of $\omega_{1}^{2} \partial_{\varphi_{1}}^{2}+2 \omega_{1}(1+\varepsilon) \partial_{\varphi_{1}} \partial_{\varphi_{2}}$ $\left(\omega_{1}=n / m\right)$ restricted to $P$ satisfy, for $\varepsilon \in \mathcal{B}_{\gamma}$,

$$
\omega_{1}\left|l_{1} \frac{\left(n l_{1}+2 l_{2} m\right)+\varepsilon 2 l_{2} m}{m}\right| \geq \frac{\omega_{1}\left|l_{1}\right| \gamma}{2\left|l_{2}\right| m^{2}} \quad \forall n l_{1}+2 m l_{2} \neq 0, l_{2} \neq 0
$$

and therefore $\bar{p} \in \mathcal{H}_{\bar{\sigma}, s+2}$ and $|\bar{p}|_{\bar{\sigma}, s+2}=O\left(|\varepsilon| m^{2} / \omega_{1}^{3} \gamma\right)$.

\section{Case B: $\omega_{1} \notin \mathbb{Q}$}

We now look for solutions of equation (1.8) when the forcing frequency $\omega_{1}$ is an irrational number.

To fix notations we shall prove Theorem B when $\int_{0}^{2 \pi} a_{2 d-1}\left(\varphi_{1}\right) d \varphi_{1}>0$ and therefore $\varepsilon>0$, i.e. $\operatorname{sign}(\varepsilon)=1$.

Fixed $0<\bar{s}<s-1 / 2$, the Nemitskii operator $u \rightarrow f\left(\varphi_{1}, u, \delta\right) \in C^{\infty}\left(B_{\rho}, \mathcal{H}_{\sigma, \bar{s}}\right)$ since, if $a_{k}\left(\varphi_{1}\right) \in H^{s}(\mathbb{T})$, then $a_{k}(\cdot) \in \mathcal{H}_{\sigma, \bar{s}}, \forall \sigma>0,0<\bar{s}<s-1 / 2$.

For $\varepsilon=0$ equation (1.8) reduces to

$$
\omega_{1} \partial_{\varphi_{1}}\left(\omega_{1} \partial_{\varphi_{1}}+2 \partial_{\varphi_{2}}\right) q=0
$$

and its solutions $q$ form, by the irrationality of $\omega_{1}$, the infinite dimensional subspace

$$
Q:=\left\{q \in \mathcal{H}_{\sigma, \bar{s}}: \partial_{\varphi_{1}} q \equiv 0\right\}=\left\{q=q\left(\varphi_{2}\right) \in H^{\sigma}(\mathbb{T})\right\} .
$$

To find solutions of (1.8) for $\varepsilon \neq 0$, we perform a Lyapunov-Schmidt reduction and we decompose the space

$$
\mathcal{H}_{\sigma, \bar{s}}=Q \oplus P
$$

where $Q \equiv H^{\sigma}(\mathbb{T})$ and

$$
P:=\left\{p=\sum_{l \in \mathbb{Z}^{2}} \hat{p}_{l} e^{\mathrm{i} l \cdot \varphi} \in \mathcal{H}_{\sigma, \bar{s}} \mid \hat{p}_{l}=0 \quad \text { for } \quad l_{1}=0\right\} .
$$

Projecting equation (1.8) onto the closed subspaces $Q$ and $P$, setting $u=q+p \in \mathcal{H}_{\sigma, \bar{s}}$ with $q \in Q, p \in P$ we obtain

$$
\begin{aligned}
(2+\varepsilon) \ddot{q}+\Pi_{Q}\left[f\left(\varphi_{1}, q+p, \delta\right)\right] & =0 \\
\mathcal{L}_{\varepsilon}[p]+\varepsilon \Pi_{P}\left[f\left(\varphi_{1}, q+p, \delta\right)\right] & =0
\end{aligned}
$$

where $\ddot{q}=\partial_{\varphi_{2}}^{2} q, \Pi_{Q}: \mathcal{H}_{\sigma, \bar{s}} \rightarrow Q$ is the projector onto $Q$,

$$
\left(\Pi_{Q} u\right)\left(\varphi_{2}\right):=\frac{1}{2 \pi} \int_{0}^{2 \pi} u\left(\varphi_{1}, \varphi_{2}\right) d \varphi_{1}
$$

and $\Pi_{P}=\mathrm{Id}-Q$ is the projector onto $P$. 
We could proceed now as in the previous section performing a finite dimensional reduction and applying variational methods. However, in this case, the infinite dimensional $(Q)$-equation can be directly solved by the Implicit Function Theorem in a space of analytic functions.

For this, it is useful to consider the parameter $\delta$ (and $\varepsilon=\delta^{2(d-1)}$ ) in the right hand side of (3.4), as an independent parameter $\delta=\eta, \varepsilon=\eta^{2(d-1)}$, and to solve the equation

$$
\mathcal{L}_{\varepsilon}[p]+\eta^{2(d-1)} \Pi_{P}\left[f\left(\varphi_{1}, q+p, \eta\right)\right]=0 \quad\left(P_{\eta}\right)
$$

for $\left(\varepsilon, \omega_{1}\right)$ in the Cantor set $\mathcal{C}_{\gamma}$ and for all $\eta$ small. In this way we highlight the smoothness of the solution $p(\eta, \varepsilon, \cdot)$ of the $\left(P_{\eta}\right)$-equation (3.5) in the variable $\eta$.

3.1. Solution of the $\left(P_{\eta}\right)$-equation. We first prove that the operator $\mathcal{L}_{\varepsilon}: P \rightarrow P$ has a bounded inverse when $\left(\varepsilon, \omega_{1}\right)$ belongs to the Cantor set $\mathcal{C}_{\gamma}$ defined in (1.6) $)$.

Lemma 3.1. For any $\varepsilon_{0}>0$ the Cantor set $\mathcal{C}_{\gamma}$ is uncountable.

Proof. Consider the set $\overline{\mathcal{C}}$ of couples $x_{1}, x_{2} \in \mathcal{B}_{\gamma}$ such that:

$$
x_{1} \in\left(-\varepsilon_{1}, \varepsilon_{1}\right), \quad x_{2} \in\left(1+\varepsilon_{1}, 2-\varepsilon_{1}\right), \quad x_{1}+x_{2} \notin \mathbb{Q}, \quad x_{1}-x_{2} \notin \mathbb{Q} .
$$

where $\varepsilon_{1}=\varepsilon_{0} / 2 . \overline{\mathcal{C}}$ is an uncountable subset of $\mathbb{R}^{2}$ since for all $x_{1} \in \mathcal{B}_{\gamma}$ the conditions $x_{1} \pm x_{2} \notin \mathbb{Q}$ exclude only a countable set of values $x_{2}$. The Lemma follows since $\mathcal{C}_{\gamma}$ contains $\psi^{-1} \overline{\mathcal{C}}$ where $\psi:\left(\varepsilon, \omega_{1}\right) \rightarrow\left(\varepsilon / \omega_{1},(2+\varepsilon) / \omega_{1}\right)$ is an invertible map for $\left(\varepsilon, \omega_{1}\right) \in\left(-\varepsilon_{0}, \varepsilon_{0}\right) \times(1,2)$.

The operator $\mathcal{L}_{\varepsilon}$ has eigenvalues $D_{l}=\left(\omega_{1} l_{1}+\varepsilon l_{2}\right)\left(\omega_{1} l_{1}+2 l_{2}+\varepsilon l_{2}\right)$.

Lemma 3.2. For $\left(\varepsilon, \omega_{1}\right) \in \mathcal{C}_{\gamma}$ the eigenvalues $D_{l}$ of $\mathcal{L}_{\varepsilon}$ restricted to $P$ satisfy

$$
\left|D_{l}\right|=\left|\left(\omega_{1} l_{1}+\varepsilon l_{2}\right)\left(\omega_{1} l_{1}+2 l_{2}+\varepsilon l_{2}\right)\right|>\gamma, \quad \forall l_{1} \neq 0 .
$$

As a consequence, the operator $\mathcal{L}_{\varepsilon}: P \rightarrow P$ has a bounded inverse $\mathcal{L}_{\varepsilon}^{-1}$ satisfying

$$
\left|\mathcal{L}_{\varepsilon}^{-1}[p]\right|_{\sigma, \bar{s}} \leq \frac{|p|_{\sigma, \bar{s}}}{\gamma}, \quad \forall p \in P
$$

Proof. Estimate (3.6) is trivially satisfied if $-l_{1} \neq \frac{\varepsilon}{\omega_{1}} l_{2}$ and $-l_{1} \neq \frac{2+\varepsilon}{\omega_{1}} l_{2}$. Now, if $-l_{1}=\left[\frac{\varepsilon}{\omega_{1}} l_{2}\right]$, then $\left|(2+\varepsilon) l_{2}+\omega_{1} l_{1}\right|>\left|(2+\varepsilon) l_{2}-\varepsilon l_{2}\right|-\frac{1}{2}>\left|l_{2}\right|$. Therefore, using $\left|\omega_{1} l_{1}+\varepsilon l_{2}\right|>\gamma /\left|l_{2}\right|$, we get (3.6). The same estimate (3.6) holds if $-l_{1}=\left[\frac{2+\varepsilon}{\omega_{1}} l_{2}\right]$ since, in this case, $\left|\omega_{1} l_{1}+\varepsilon l_{2}\right|>\left|(2+\varepsilon) l_{2}-\varepsilon l_{2}\right|-\frac{1}{2}>\left|l_{2}\right|$.

Fixed points of the nonlinear operator $\mathcal{G}: P \rightarrow P$ defined by

$$
\mathcal{G}(\eta, p):=-\eta^{2(d-1)} \mathcal{L}_{\varepsilon}^{-1} \Pi_{P} f\left(\varphi_{1}, q+p, \eta\right)
$$

are solutions of the $\left(P_{\eta}\right)$-equation. 
Lemma 3.3. Assume $\left(\varepsilon, \omega_{1}\right) \in \mathcal{C}_{\gamma} . \forall R>0$ there exists $\eta_{0}(R), C_{0}(R)>0$ such that $\forall|q|_{H^{\sigma}(\mathbb{T})} \leq R, 0<\eta \gamma^{-c} \leq \eta_{0}(R)$, with $c=1 / 2(d-1)$, there exists a unique $p(\eta, q) \in P \cap \mathcal{H}_{\sigma, \bar{s}}$ solving the $\left(P_{\eta}\right)$-equation (3.5) and satisfying

$$
|p(\eta, q)|_{\sigma, \bar{s}} \leq C_{0}(R) \eta^{2(d-1)} \gamma^{-1}
$$

and the equivariance property

$$
p\left(\eta, q_{\theta}\right)\left(\varphi_{1}, \varphi_{2}\right)=p(\eta, q)\left(\varphi_{1}, \varphi_{2}-\theta\right), \quad \forall \theta \in \mathbb{T}
$$

where $q_{\theta}\left(\varphi_{1}, \varphi_{2}\right):=q\left(\varphi_{1}, \varphi_{2}-\theta\right)$. Moreover $p(\cdot, \cdot) \in C^{1}\left(\left(0, \eta_{0}(R)\right) \times Q ; P\right)$.

Proof. In the Appendix.

3.2. The $(Q)$-equation. Once the $\left(P_{\eta}\right)$-equation has been solved by $p(\eta, q) \in P$ there remains the infinite dimensional bifurcation equation

$$
(2+\varepsilon) \ddot{q}+\Pi_{Q}\left[f\left(\varphi_{1}, q+p(\eta, q), \eta\right)\right]=0 .
$$

Recalling (1.9), the $(Q)$-equation (3.10) evaluated at $\eta=0$ reduces to the ordinary differential equation

$$
(2+\varepsilon) \ddot{q}+\left\langle a_{2 d-1}\right\rangle q^{2 d-1}=0
$$

where $\left\langle a_{2 d-1}\right\rangle:=(1 / 2 \pi) \int_{0}^{2 \pi} a_{2 d-1}\left(\varphi_{1}\right) d \varphi_{1}$.

Equation (3.11) is a superlinear autonomous Hamiltonian system with one degree of freedom and can be studied by a direct phase-space analysis.

Lemma 3.4. There exists $\bar{\sigma}>0$ such that, equation (3.11) possesses a $2 \pi$-periodic, analytic solution $\bar{q}\left(\varphi_{2}\right) \in H^{\bar{\sigma}}(\mathbb{T})$. Morevoer, $\bar{q}\left(\varphi_{2}\right)$ is non-degenerate up to time translations, i.e. the linearized equation on $\bar{q}$

$$
(2+\varepsilon) \ddot{h}+(2 d-1)\left\langle a_{2 d-1}\right\rangle \bar{q}^{2(d-1)} h=0
$$

possesses a one-dimensional space of $2 \pi$-periodic solutions, spanned by $\dot{\bar{q}}$.

Proof. Up to a rescaling, equation (3.11) can be written as $\ddot{x}=-V^{\prime}(x)$ with potential energy $V(x):=x^{2 d}$. All solutions of such system are analytic and periodic with period

$$
T(E)=4 \int_{0}^{E^{\frac{1}{2 d}}} \frac{d x}{\sqrt{2\left(E-x^{2 d}\right)}}=4 E^{\frac{1}{2 d}-\frac{1}{2}} \int_{0}^{1} \frac{d x}{\sqrt{2\left(1-x^{2 d}\right)}} .
$$

The equation $T(E)=2 \pi$ has a solution $\bar{q}\left(\varphi_{2}\right)$ which is in $H^{\bar{\sigma}}(\mathbb{T})$ for some appropriate $\bar{\sigma}>0$. The non-degeneracy of the corresponding $2 \pi$-periodic solution follows by

$$
\frac{d T}{d E}=2\left(\frac{1}{d}-1\right) E^{\frac{1}{2 d}-\frac{3}{2}} \int_{0}^{1} \frac{d x}{\sqrt{2\left(1-x^{2 d}\right)}} \neq 0
$$

and the next Proposition proved in the Appendix. 
Proposition 2. Suppose the autonomous second order equation $-\ddot{x}=V^{\prime}(x), x \in \mathbb{R}$, possesses a continuous family of non-constant periodic solutions $x(E, t)$ with energy $E$ and period $T(E)$ satisfying the anysocronicity condition $\frac{d T(E)}{d E} \neq 0$. Then $x(E, t)$ is nondegenerate up to time translations, i.e. the $T(E)$-periodic solutions of the linearized equation

$$
-\ddot{h}=D^{2} V(x(E, t)) h
$$

form a one dimensional subspace spanned by $\left(\partial_{t} x\right)(E, t)$.

From now on we fix $\bar{R}:=|\bar{q}|_{H^{\bar{\sigma}(\mathbb{T})}}+1$ in Lemma 3.3 and take $0<\eta \gamma^{-c} \leq \eta_{0}(\bar{R})$.

By Lemma 3.4 and (3.9), we can construct solutions of the infinite dimensional bifurcation equation (3.10) by means of the Implicit Function Theorem:

Lemma 3.5. There exist $0<\eta_{1} \leq \eta_{0}(\bar{R}), C_{1}>0$ such that for all $0<\eta \gamma^{-c} \leq \eta_{1}$, equation (3.10) has a unique (up to translations) solution $\bar{q}_{\eta}\left(\varphi_{2}\right) \in H^{\bar{\sigma}}(\mathbb{T})$ satisfying

$$
\left|\bar{q}_{\eta}-\bar{q}\right|_{H^{\bar{\sigma}}(\mathbb{T})} \leq C_{1}|\eta|
$$

Proof of Theorem B. Setting again $\delta \equiv \eta, \bar{q}_{\varepsilon}\left(\varphi_{2}\right)+p\left(\varepsilon, \bar{q}_{\varepsilon}\right)$ solves (1.8) and

$$
u(\varepsilon, \varphi)=|\varepsilon|^{1 / 2(d-1)}\left[\bar{q}_{\varepsilon}\left(\varphi_{2}\right)+p\left(\varepsilon, \bar{q}_{\varepsilon}\right)\right]
$$

is a non trivial solution of (1.4). The bound (1.7) follows by (3.8). As in Theorem A the solution $u$ depends on both the variables $\left(\varphi_{1}, \varphi_{2}\right)$. Finally, the solution $v(\varepsilon, t, x):=$ $u\left(\varepsilon, \omega_{1} t, x+\omega_{2} t\right)$ of (1.1) is quasi-periodic since, by the definition of $\mathcal{C}_{\gamma}, \omega_{1} / \omega_{2}=\omega_{1} /$ $(1+\varepsilon) \notin \mathbb{Q}$.

Remark 3. To prove existence of solutions of (1.8), i.e. (1.1), it is sufficient that the second order equation (3.11) possessess a continuous, nonisocronous family of nonconstant periodic orbits one of them having period $2 \pi / j$, see Proposition 2 .

The hypothesys that the leading term in the nonlinearity $f$ is an odd power of $u$ is not of technical nature. The following non-existence result holds:

Proposition 3. (Non-existence) Let $f\left(\varphi_{1}, u\right)=a\left(\varphi_{1}\right) u^{D}$ with $D$ even and $\int_{0}^{2 \pi} a\left(\varphi_{1}\right)$ $d \varphi_{1} \neq 0 . \forall R>0$, there exists $\varepsilon_{0}>0$ such that $\forall \sigma \geq 0, \bar{s}>s-\frac{1}{2}, \forall\left(\varepsilon, \omega_{1}\right) \in \mathcal{C}_{\gamma}$ with $|\varepsilon|<\varepsilon_{0}$, equation (1.4) does not possess solutions $u \in \mathcal{H}_{\sigma, \bar{s}}$ in the ball $|u|_{\sigma, \bar{s}} \leq$ $R|\varepsilon|^{1 /(D-1)}$.

Proof. We first rescale equation (1.4) with $u \rightarrow|\varepsilon|^{1 /(D-1)} u$ obtaining

$$
\mathcal{L}_{\varepsilon} u+|\varepsilon| a\left(\varphi_{1}\right) u^{D}=0 .
$$

Write any solution $u_{\varepsilon} \in B_{\sigma, \bar{s}}(R):=\left\{u \in \mathcal{H}_{\sigma, \bar{s}}:|u|_{\sigma, \bar{s}} \leq R\right\}$ of (3.14) as $u_{\varepsilon}=q_{\varepsilon}+p_{\varepsilon}$ with $q_{\varepsilon} \in Q, p_{\varepsilon} \in P$. $p_{\varepsilon}$ satisfies the $(P)$-equation $\mathcal{L}_{\varepsilon} p+|\varepsilon| \Pi_{P} a\left(\varphi_{1}\right) u^{D}=0$ and therefore $\left|p_{\varepsilon}\right|_{\sigma, \bar{s}} \leq C(R)|\varepsilon|$. Then, for $\varepsilon$ small enough, $p_{\varepsilon} \equiv p\left(\varepsilon, q_{\varepsilon}\right)$ where $p\left(\varepsilon, q_{\varepsilon}\right)$ is constructed as in Lemma 3.3 and satisfies the estimate $\left|p\left(\varepsilon, q_{\varepsilon}\right)\right|_{\sigma, \bar{s}} \leq C|\varepsilon|\left|q_{\varepsilon}\right|_{H^{\sigma}(\mathbb{T})}^{D}$. 
The projection $q_{\varepsilon}$ satisfies the $(Q)$-equation

$$
(2+\varepsilon) \ddot{q}_{\varepsilon}+\operatorname{sign}(\varepsilon) \Pi_{Q}\left[a\left(\varphi_{1}\right)\left(q_{\varepsilon}+p\left(\varepsilon, q_{\varepsilon}\right)\right)^{D}\right]=0
$$

and therefore $\left|q_{\varepsilon}\right|_{H^{\sigma, 2}(\mathbb{T})} \leq C(R)$.

We claim that $q_{\varepsilon} \rightarrow 0$ in $H^{\sigma}(\mathbb{T})$ (and so in $\mathcal{H}_{\sigma, \bar{s}}$ ) for $\varepsilon \rightarrow 0$. Indeed, from any subsequence $q_{\varepsilon}$, we can extract by the compact embedding $H^{\sigma, 2}(\mathbb{T}) \hookrightarrow H^{\sigma}(\mathbb{T})$ another convergent subsequence $q_{\varepsilon_{n}}$ such that $q_{\varepsilon_{n}} \rightarrow \bar{q} \in H^{\sigma}(\mathbb{T})$. By (3.15), we deduce that

$$
2 \ddot{\bar{q}}+\operatorname{sign}(\varepsilon)\langle a\rangle \bar{q}^{D}=0
$$

where $\langle a\rangle:=\int_{0}^{2 \pi} a\left(\varphi_{1}\right) d \varphi_{1} \neq 0$. Such equation does not possess non-trivial periodic solutions for both $\operatorname{sign}(\varepsilon)= \pm 1$, i.e. $\varepsilon>0$ and $\varepsilon<0$, and we conclude that $\bar{q}=0$.

We finally prove that equation (3.15) does not possess non-trivial periodic solutions in a small neighborhood of the origin.

Linearizing equation (3.15) at $q=0$ we get $(2+\varepsilon) \ddot{h}=0$ whose solutions in $H^{\sigma}(\mathbb{T})$ are the constants. We can perform another Lyapunov-Schmidt reduction close to 0 decomposing $H^{\sigma}(\mathbb{T})=\{$ constants $\} \oplus$ zero average functions $\}$, namely $q_{\varepsilon}=\rho+w$. By the Implicit function Theorem we get that for any constant $|\rho| \leq \rho_{0}$ small enough (independently of $\varepsilon$ ) there exists a unique zero average function $w_{\rho}$ with $\left|w_{\rho}\right|_{H^{\sigma}(\mathbb{T})}=$ $O\left(\rho^{D}\right)$ solving

$$
(2+\varepsilon) \ddot{w}_{\rho}+\left[a\left(\varphi_{1}\right)\left(\rho+w_{\rho}+p\left(\varepsilon, q_{\varepsilon}\right)\right)^{D}-\left\langle a\left(\varphi_{1}\right)\left(\rho+w_{\rho}+p\left(\varepsilon, q_{\varepsilon}\right)\right)^{D}\right\rangle\right]=0 .
$$

Hence $\rho$ is such that

$$
0=\left\langle a\left(\varphi_{1}\right)\left(\rho+w_{\rho}+p\left(\varepsilon, q_{\varepsilon}\right)\right)^{D}\right\rangle=\langle a\rangle \rho^{D}+o\left(\rho^{D}\right) .
$$

This implies $\rho=0$ since $\langle a\rangle \neq 0$ and so $q_{\varepsilon}=\rho+w_{\rho}=0$.

\section{ApPendix}

Lemma 4.1. $\mathcal{H}_{\sigma, s}$ is a Banach algebra for $\sigma, s \geq 0$.

Proof. By the product Cauchy formula

$$
u v=\sum_{j \in \mathbb{Z}^{2}}\left(\sum_{k \in \mathbb{Z}^{2}} u_{j-k} v_{k}\right) e^{\mathrm{i} j \cdot \varphi}
$$

and therefore

$$
\begin{aligned}
|u v|_{\sigma, s} & :=\sum_{j \in \mathbb{Z}^{2}} e^{\sigma\left|j_{2}\right|}\left[j_{1}\right]^{s}\left|\sum_{k \in Z^{2}} u_{j-k} v_{k}\right| \leq \sum_{j \in \mathbb{Z}^{2}} e^{\sigma\left|j_{2}\right|}\left[j_{1}\right]^{s} \sum_{k \in \mathbb{Z}^{2}}\left|u_{j-k}\right|\left|v_{k}\right| \\
& \leq \sum_{k \in \mathbb{Z}^{2}}\left|v_{k}\right| \sum_{j \in \mathbb{Z}^{2}}\left|u_{j-k}\right| e^{\sigma\left|j_{2}\right|}\left[j_{1}\right]^{s} \\
& \leq 2^{s} \sum_{k \in \mathbb{Z}^{2}}\left|v_{k}\right| e^{\sigma\left|k_{2}\right|}\left[k_{1}\right]^{s} \sum_{j \in \mathbb{Z}^{2}}\left|u_{j-k}\right| e^{\sigma\left|j_{2}-k_{2}\right|}\left[j_{1}-k_{1}\right]^{s}:=2^{s}|u|_{\sigma, s}|v|_{\sigma, s}
\end{aligned}
$$

since $e^{\sigma\left|j_{2}\right|} \leq e^{\sigma\left|j_{2}-k_{2}\right|} e^{\sigma\left|k_{2}\right|}$ and $\left[j_{1}\right] \leq 2\left[j_{1}-k_{1}\right]\left[k_{1}\right]$ for all $k, j \in \mathbb{Z}^{2}$. 
Proof of Lemma 2.3, Let us consider

$$
B:=\left\{\left(q_{2}, p\right) \in Q_{2} \oplus P:\left|q_{2}\right|_{\sigma, s} \leq \rho_{1},|p|_{\sigma, s} \leq \rho_{2}\right\}
$$

with norm $\left|\left(q_{2}, p\right)\right|_{\sigma, s}:=\left|q_{2}\right|_{\sigma, s}+|p|_{\sigma, s}$. We claim that, under the assumptions (2.9) there exists $0<\rho_{1}, \rho_{2}<1$, see (4.6) , such that the map $\left(q_{2}, p\right) \rightarrow \mathcal{G}\left(q_{2}, p ; q_{1}\right)$ is a contraction in $B$, i.e.:

(i) $\left(q_{2}, p\right) \in B \Longrightarrow \mathcal{G}\left(q_{2}, p ; q_{1}\right) \in B$;

(ii) $\left|\mathcal{G}\left(q_{2}, p ; q_{1}\right)-\mathcal{G}\left(\widetilde{q}_{2}, \widetilde{p} ; q_{1}\right)\right|_{\sigma, s} \leq(1 / 2)\left|\left(q_{2}, p\right)-\left(\widetilde{q_{2}}, \widetilde{p}\right)\right|_{\sigma, s}, \quad \forall\left(q_{2}, p\right),\left(\widetilde{q}_{2}, \widetilde{p}\right) \in B$.

In the following $\kappa_{i}$ will denote positive constants independent on $R, N$ and $\varepsilon$ (i.e. on $\left.\delta:=|\varepsilon|^{1 / 2(d-1)}\right)$.

By (2.7) and the Banach algebra property of $\mathcal{H}_{\sigma, s}$

$$
\begin{aligned}
\left|\mathcal{G}_{1}\left(q_{2}, p ; q_{1}\right)\right|_{\sigma, s} & =\left|L_{1}^{-1} \Pi_{Q_{2}} f\left(\varphi_{1}, q_{1}+q_{2}+p, \delta\right)\right|_{\sigma, s} \\
& \leq \frac{\kappa_{1}}{N^{2}}\left(\left|q_{1}\right|_{\sigma, s}^{2 d-1}+\left|q_{2}\right|_{\sigma, s}^{2 d-1}+|p|_{\sigma, s}^{2 d-1}\right)
\end{aligned}
$$

provided that $0 \leq \delta \leq \delta_{0}(R)$. Similarly, for $\varepsilon \in \mathcal{B}_{\gamma}$, by (2.6),

$$
\begin{aligned}
\left|\mathcal{G}_{2}\left(q_{2}, p ; q_{1}\right)\right|_{\sigma, s} & =\left|\varepsilon \mathcal{L}_{\varepsilon}^{-1} \Pi_{P} f\left(\varphi_{1}, q_{1}+q_{2}+p, \delta\right)\right|_{\sigma, s} \\
& \leq \kappa_{2}|\varepsilon| \gamma^{-1}\left(\left|q_{1}\right|_{\sigma, s}^{2 d-1}+\left|q_{2}\right|_{\sigma, s}^{2 d-1}+|p|_{\sigma, s}^{2 d-1}\right) .
\end{aligned}
$$

For all $q_{1} \in Q_{1}(N)$ and since $0 \leq s<1 / 2$

$$
\begin{aligned}
\left|q_{1}\right|_{\sigma, s} & =\sum_{\left|l_{2}\right| \leq N}\left|\hat{q}_{0, l_{2}}\right| e^{\sigma\left|l_{2}\right|}+\left|\hat{q}_{-2 l_{2}, l_{2}}\right| e^{\sigma\left|l_{2}\right|}\left[-2 l_{2}\right]^{s} \\
& \leq e^{\sigma N} \sum_{\left|l_{2}\right| \leq N}\left|\hat{q}_{0, l_{2}}\right|+\left|\hat{q}_{-2 l_{2}, l_{2}}\right|\left[-2 l_{2}\right]^{s} \leq \kappa_{3}\left[\left(\sum_{\left|l_{2}\right| \leq N}\left|\hat{q}_{0, l_{2}}\right|^{2}\left[l_{2}\right]^{2}\right)^{1 / 2}\left(\sum_{l_{2} \in \mathbb{Z}} \frac{1}{\left[l_{2}\right]^{2}}\right)^{1 / 2}\right. \\
(4.3) & \left.+\left(\sum_{\left|l_{2}\right| \leq N}\left|\hat{q}_{-2 l_{2}, l_{2}}\right|^{2}\left[l_{2}\right]^{2}\right)^{1 / 2}\left(\sum_{l_{2} \in \mathbb{Z}} \frac{1}{\left[l_{2}\right]^{2(1-s)}}\right)^{1 / 2}\right] \leq \kappa_{4}\left|q_{1}\right|_{H^{1}}
\end{aligned}
$$

whenever $0 \leq \sigma N \leq 1$.

Substituting in (4.1)-(4.2) we get $\forall\left|q_{1}\right|_{H^{1}} \leq 2 R, \forall\left|q_{2}\right|_{\sigma, s} \leq \rho_{1}, \forall|p|_{\sigma, s} \leq \rho_{2}$

$$
\begin{aligned}
\left|\mathcal{G}_{1}\left(q_{2}, p ; q_{1}\right)\right|_{\sigma, s} & \leq \kappa_{5} N^{-2}\left(R^{2 d-1}+\rho_{1}^{2 d-1}+\rho_{2}^{2 d-1}\right) \\
\left|\mathcal{G}_{2}\left(q_{2}, p ; q_{1}\right)\right|_{\sigma, s} & \leq \kappa_{5}|\varepsilon| \gamma^{-1}\left(R^{2 d-1}+\rho_{1}^{2 d-1}+\rho_{2}^{2 d-1}\right) .
\end{aligned}
$$

Now, setting $C_{0}(R):=\kappa_{5} R^{2 d-1}$, we define

$$
\rho_{1}:=\frac{2 C_{0}(R)}{N^{2}} \quad \rho_{2}:=2|\varepsilon| \gamma^{-1} C_{0}(R) .
$$

By (4.4), (4.5) there exists $N_{0}(R) \in \mathbb{N}^{+}$and $\varepsilon_{0}(R)>0$ such that $\forall N \geq N_{0}(R)$ and $\forall|\varepsilon| \gamma^{-1} \leq \varepsilon_{0}(R)$

$$
\left|\mathcal{G}_{1}\left(q_{2}, p ; q_{1}\right)\right|_{\sigma, s} \leq \rho_{1} \quad\left|\mathcal{G}_{2}\left(q_{2}, p ; q_{1}\right)\right|_{\sigma, s} \leq \rho_{2}
$$

proving (i). Item (ii) is obtained with similar estimates.

By the Contraction Mapping Theorem there exists a unique fixed point $\left(q_{2}\left(q_{1}\right), p\left(q_{1}\right)\right):=$ $\left(q_{2}\left(\varepsilon, N, q_{1}\right), p\left(\varepsilon, N, q_{1}\right)\right)$ of $\mathcal{G}$ in $B$. The bounds (2.10) follow by (4.6). 
Since $\mathcal{G} \in C^{1}\left(Q_{2} \oplus P \times Q_{1} ; Q_{2} \oplus P \times Q_{1}\right)$ the Implicit function Theorem implies that the maps $Q_{1} \ni q_{1} \rightarrow\left(q_{2}\left(\varepsilon, N, q_{1}\right), p\left(\varepsilon, N, q_{1}\right)\right)$ are $C^{1}$.

Differentiating $\left(q_{2}\left(q_{1}\right), p\left(q_{1}\right)\right)=\mathcal{G}\left(q_{2}\left(q_{1}\right), p\left(q_{1}\right), q_{1}\right)$

$$
\begin{array}{r}
q_{2}^{\prime}\left(q_{1}\right)[h]=-L_{1}^{-1} \Pi_{Q_{2}}\left(\partial_{u} f\right)\left(\varphi_{1}, q_{1}+q_{2}\left(q_{1}\right)+p\left(q_{1}\right), \delta\right)\left(h+q_{2}^{\prime}\left(q_{1}\right)[h]+p^{\prime}\left(q_{1}\right)[h]\right) \\
p^{\prime}\left(q_{1}\right)[h]=-\varepsilon \mathcal{L}_{\varepsilon}^{-1} \Pi_{Q_{2}}\left(\partial_{u} f\right)\left(\varphi_{1}, q_{1}+q_{2}\left(q_{1}\right)+p\left(q_{1}\right), \delta\right)\left(h+q_{2}^{\prime}\left(q_{1}\right)[h]+p^{\prime}\left(q_{1}\right)[h]\right)
\end{array}
$$

and using (2.7), (2.6) and the Banach algebra property of $\mathcal{H}_{\sigma, s}$

$$
\begin{aligned}
& \left|q_{2}^{\prime}\left(q_{1}\right)[h]\right|_{\sigma, s} \leq C(R) N^{-2}\left(|h|_{\sigma, s}+\left|q_{2}^{\prime}\left(q_{1}\right)[h]\right|_{\sigma, s}+\left|p^{\prime}\left(q_{1}\right)[h]\right|_{\sigma, s}\right) \\
& \left|p^{\prime}\left(q_{1}\right)[h]\right|_{\sigma, s} \leq C(R)|\varepsilon| \gamma^{-1}\left(|h|_{\sigma, s}+\left|q_{2}^{\prime}\left(q_{1}\right)[h]\right|_{\sigma, s}+\left|p^{\prime}\left(q_{1}\right)[h]\right|_{\sigma, s}\right)
\end{aligned}
$$

which implies the bounds (2.11) since

$$
\operatorname{det}\left|\begin{array}{ll}
1-C(R) N^{-2} & -C(R) N^{-2} \\
-C(R)|\varepsilon| \gamma^{-1} & 1-C(R)|\varepsilon| \gamma^{-1}
\end{array}\right| \geq \frac{1}{2}
$$

for $C(R)\left(|\varepsilon| \gamma^{-1}+N^{-2}\right)$ small enough and (4.3).

Proof of Lemma 2.4 By (2.4), (2.5) we have that, at $u:=q_{1}+q_{2}\left(q_{1}\right)+p\left(q_{1}\right)$,

$$
d \Psi_{\varepsilon}(u)[h]=0 \quad \forall h \in Q_{2} \quad \text { and } \quad d \Psi_{\varepsilon}(u)[h]=0 \quad \forall h \in P .
$$

Since $q_{2}^{\prime}\left(q_{1}\right)[k] \in Q_{2}$ and $p^{\prime}\left(q_{1}\right)[k] \in P \forall k \in Q_{1}$, we deduce

$$
d \Phi_{\varepsilon, N}\left(q_{1}\right)[k]=d \Psi_{\varepsilon}(u)\left[h+q_{2}^{\prime}\left(q_{1}\right)[k]+p^{\prime}\left(q_{1}\right)[k]\right]=d \Psi_{\varepsilon}(u)[k] \quad \forall k \in Q_{1}
$$

and therefore $u:=q_{1}+p\left(q_{1}\right)+q_{2}\left(q_{1}\right)$ solves also the $\left(Q_{1}\right)$-equation (2.3).

Write $\Psi_{\varepsilon}(u)=\Psi_{\varepsilon}^{(2)}(u)-\varepsilon \int_{\mathbb{T}^{2}} F\left(\varphi_{1}, u, \delta\right)$ where

$$
\Psi_{\varepsilon}^{(2)}(u):=\int_{\mathbb{T}^{2}} \frac{1}{2}\left(\partial_{\varphi_{1}} u\right)^{2}+(1+\varepsilon)\left(\partial_{\varphi_{1}} u\right)\left(\partial_{\varphi_{2}} u\right)+\frac{\varepsilon(2+\varepsilon)}{2}\left(\partial_{\varphi_{2}} u\right)^{2}
$$

is an homogeneous functional of degree two. By homogeneity:

$$
\Psi_{\varepsilon}(u)=\frac{1}{2} d \Psi_{\varepsilon}^{(2)}(u)[u]-\varepsilon \int_{\mathbb{T}^{2}} F\left(\varphi_{1}, u, \delta\right) .
$$

By (2.4), (2.5) (i.e. (4.7))

$$
d \Psi_{\varepsilon}^{(2)}\left(q_{1}+q_{2}\left(q_{1}\right)+p\left(q_{1}\right)\right)\left[q_{2}\left(q_{1}\right)+p\left(q_{1}\right)\right]=\varepsilon \int_{\mathbb{T}^{2}} f\left(\varphi_{1}, u, \delta\right)\left(q_{2}\left(q_{1}\right)+p\left(q_{1}\right)\right) .
$$


Substituting in (4.8) we obtain, at $u=q_{1}+q_{2}\left(q_{1}\right)+p\left(q_{1}\right)$

$$
\begin{aligned}
\Phi_{\varepsilon, N}\left(q_{1}\right) & =\Psi_{\varepsilon}\left(q_{1}+p\left(q_{1}\right)+q_{2}\left(q_{1}\right)\right)=\frac{1}{2} d \Psi_{\varepsilon}^{(2)}(u)\left[q_{1}+p\left(q_{1}\right)+q_{2}\left(q_{1}\right)\right]-\varepsilon \int_{\mathbb{T}^{2}} F\left(\varphi_{1}, u, \delta\right) \\
& =\frac{1}{2} d \Psi_{\varepsilon}^{(2)}\left(q_{1}\right)\left[q_{1}\right]-\varepsilon \int_{\mathbb{T}^{2}} F\left(\varphi_{1}, u, \delta\right)+\frac{1}{2} f\left(\varphi_{1}, u, \delta\right)\left(q_{2}\left(q_{1}\right)+p\left(q_{1}\right)\right) \\
& =\Psi_{0}\left(q_{1}\right)+\varepsilon \int_{\mathbb{T}^{2}} \frac{(2+\varepsilon)}{2}\left(\partial_{\varphi_{2}} q_{1}\right)^{2}+\left(\partial_{\varphi_{1}} q_{1}\right)\left(\partial_{\varphi_{2}} q_{1}\right)-F\left(\varphi_{1}, u, \delta\right) \\
& +\frac{1}{2} f\left(\varphi_{1}, u, \delta\right)\left(q_{2}\left(q_{1}\right)+p\left(q_{1}\right)\right)=\mathrm{const}+\varepsilon\left(\Gamma\left(q_{1}\right)+\mathcal{R}_{\varepsilon, N}\left(q_{1}\right)\right)
\end{aligned}
$$

because $\Psi_{0}\left(q_{1}\right) \equiv$ const.

By (2.10) the bounds (2.14) $-(2.15)$ follow.

Proof of Lemma 3.3. The existence of $p(\eta, q) \in \mathcal{H}_{\sigma, \bar{s}}$ can be proved as in Lemma 2.3 using the Contraction Mapping Theorem. The smoothness of $p(\eta, q)$ follows by the Implicit Function Theorem since $\mathcal{G}(\eta, p)$ is smooth in $\eta$ and $q$.

By the invariance of equation (3.5) under translations in the $\varphi_{2}$ variable the function $p(\eta, q)\left(\varphi_{1}, \varphi_{2}-\theta\right)$ solves

$$
p(\eta, q)\left(\varphi_{1}, \varphi_{2}-\theta\right)+\eta^{2(d-1)} \mathcal{L}_{\varepsilon}^{-1} \Pi_{P} f\left(\varphi_{1}, q_{\theta}+p(\eta, q)\left(\varphi_{1}, \varphi_{2}-\theta\right), \eta\right)=0
$$

and, therefore, by uniqueness (3.9) holds.

Proof of Proposition 2 Write $x(E, t)=y(\omega(E) t, E)$ where $y(\varphi, E)$ is $2 \pi$-periodic in $\varphi$ and $\omega(E):=2 \pi / T(E)$. The functions $\left(\partial_{t} x\right)(E, t)$ and

$$
\left(\partial_{E} x\right)(E, t)=t \frac{d \omega(E)}{d E}\left(\partial_{\varphi} y\right)(\omega(E) t, E)+\left(\partial_{E} y\right)(\omega(E) t, E)
$$

are two linearly independent solutions of the linearized equation (3.13).$\left(\partial_{t} x\right)(E, t)$ is $2 \pi$-periodic while, since

$$
\frac{d \omega(T)}{d T}=2 \pi T(E)^{-2} \frac{d E(T)}{d T} \neq 0 \quad \text { and } \quad\left(\partial_{\varphi} y\right)(\varphi, E) \not \equiv 0
$$

(if not $x(E, t)$ would be constant in $t),\left(\partial_{E} x\right)(E, t)$ is not $2 \pi$-periodic. We conclude that the space of $T(E)$-periodic solutions of (3.13) form a 1-dimensional linear space spanned by $\left(\partial_{t} x\right)(E, t)$.

\section{REFERENCES}

[AB] A. Ambrosetti, M. Badiale, Homoclinics: Poincaré-Melnikov type results via a variational approach, Annales I. H. P. - Analyse nonlin., vol. 15, n.2, 1998, p. 233-252.

[BP] D. Bambusi, S. Paleari, Families of periodic solutions of resonant PDE's, J. Nonlinear Sci. 11 (2001), no. 1, 69-87.

[BaB] D. Bambusi, M. Berti, A Birkhoof-Lewis type theorem for some Hamiltonian PDE's, to appear on SIAM Journal on Mathematical Analysis.

[BR] V. Benci, P. Rabinowitz, Critical point theorems for indefinite functionals, Invent. Math. 52 (1979), no. 3, 241-273.

[BBi] M. Berti, L. Biasco, Periodic solutions of nonlinear wave equations with non-monotone forcing terms, to appear on Rend. Mat. Acc. Naz. Lincei. 
[BBi1] M. Berti, L. Biasco, Forced vibrations of wave equations with non-monotone nonlinearities, Preprint Sissa 2004.

[BBV] M. Berti, L. Biasco, E. Valdinoci, Periodic orbits close to elliptic tori and applications to the three-body problem, Annali Scuola Normale Sup. di Pisa, Cl. Sci. (V) 3, 87-138, 2004.

[BB1] M. Berti, P. Bolle, Periodic solutions of nonlinear wave equations with general nonlinearities, Comm. Math. Phys. 243 (2003), no. 2, 315-328.

[BB2] M. Berti, P. Bolle, Multiplicity of periodic solutions of nonlinear wave equations, Nonlinear Anal., no. 56 (2004), 1011-1046.

[BB3] M. Berti, P. Bolle, Cantor families of periodic solutions for completely resonant non linear wave equations, preprint SISSA (2004).

[B1] J. Bourgain, Quasi-periodic solutions of Hamiltonian perturbations of 2D linear Schrödinger equations, Ann. of Math. (2) 148 (1998), no. 2, 363-439.

[B2] J. Bourgain, Periodic solutions of nonlinear wave equations, Chicago Lectures in Math., Univ. Chicago Press, Chicago, IL, 1999.

[BN] H. Brezis, L. Nirenberg, Forced vibrations for a nonlinear wave equation, Comm. Pure Appl. Math. 31 (1978), no. 1, 1-30.

[C] J. M. Coron, Periodic solutions of a nonlinear wave equation without assumption of monotonicity, Math. Ann. 262 (1983), no. 2, 273-285.

[CW] W. Craig, E. Wayne, Newton's method and periodic solutions of nonlinear wave equations, Comm. Pure Appl. Math. 46 (1993), 1409-1498.

[GMP] G. Gentile, V. Mastropietro, M. Procesi, Periodic solutions of completely resonant nonlinear wave equations, to appear in Comm. Math. Phys.

[GP] G. Gentile, M. Procesi, Conservation of resonant periodic solutions for the one-dimensional nonlinear Schrödinger equation, preprint.

[Mc] P.J. McKenna, On solutions of a nonlinear wave question when the ratio of the period to the length of the interval is irrational, Proc. Amer. Math. Soc. 93 (1985), no. 1, 59-64.

[K] S.B. Kuksin, Analysis of Hamiltonian PDEs, Oxford Lecture Series in Mathematics and its Applications, 19. Oxford University Press, 2000.

[LS] B.V. Lidskiu, E.I. Shul'man, Periodic solutions of the equation $u_{t t}-u_{x x}+u^{3}=0$, Funct. Anal. Appl. 22 (1988), no. 4, 332-333.

[PY] P.I. Plotinikov, L.N. Yungermann, Periodic solutions of a weakly nonlinear wave equation with an irrational relation of period to interval length, transl. in Diff. Eq. 24 (1988) n. 9 pp. 1059-1065.

[P] J. Pöschel, Quasi-periodic solutions for a nonlinear wave equation, Comment. Math. Helv. 71 (1996), no. 2, 269-296.

[P1] M. Procesi, Quasi-periodic solutions for completely resonant wave equations in $1 D$ and $2 D$, to appear in Discr. Cont. Dyn. Syst.

[P2] M. Procesi, Families of quasi-periodic solutions for a completely resonant wave equation, preprint.

[R1] P. Rabinowitz, Periodic solutions of nonlinear hyperbolic partial differential equations, Comm. Pure Appl. Math., 201967 145-205.

[R2] P. Rabinowitz, Time periodic solutions of nonlinear wave equations, Manuscripta Math. 5 (1971), 165-194.

[R3] P. H. Rabinowitz, Minimax methods in critical point theory with applications to differential equations, CBMS Regional Conference Series in Mathematics, 65. American Mathematical Society, 1986.

[Wa] C.E. Wayne, Periodic and quasi-periodic solutions of nonlinear wave equations via KAM theory, Comm. Math. Phys. 127 (1990), no. 3, 479-528. 